\title{
MÉMOIRE SUR L'ÉLIMINATION
}

\section{PAR}

\section{J. HADAMARD}

a BORDEAUX.

I. La méthode des fonctions symétriques apprend à éliminer les inconnues $x_{1}, x_{2}, \ldots, x_{n}$ entre les équations

$$
f_{1}=0, \quad f_{2}=0, \cdots, f_{n+1}=0
$$

en formant le produit $\Pi f_{n+1}\left(x_{1}, x_{2}, \ldots, x_{n}\right)$; étendu aux systèmes de valeurs de $x_{1}, x_{2}, \ldots, x_{n}$ qui vérifient les $n$ premières équations (I).

On obtient ainsi pour le résultant $n+I$ expressions différentes en considérant successivement comme la dernière chacune des équations données. Il peut être utile de savoir comparer entre elles ces différentes expressions, ou plutôt leurs numérateurs. Cette comparaison peut même se présenter comme nécessaire dans certaines méthodes d'élimination (voir, par exemple, Otro Brermann, Über die Bildung der Eliminanten eines Systems algebraischer Gleichungen ${ }^{1}$ ).

Comme on arrive à des résultats intéressant différents points de la théorie de l'élimination, nous sommes amenés à reprendre l'ensemble de cette théorie, après quoi nous aurons à présenter certaines applications géométriques. Vienne 1894.

Monatshefte für Mathematik und Physik, $5^{\ominus}$ année, pag. I7-33; 


\section{I.}

2. Rappelons d'abord ce qui se passe pour le cas d'une seule inconnue. Soient les deux équations $f_{1}(x)=0, f_{2}(x)=0$, ou, en rendant homogène,

$$
f_{1}\left(x_{1}, x_{2}\right)=0, \quad f_{2}\left(x_{1}, x_{2}\right)=0
$$

de degrés $m_{1}, m_{2}$ respectivement. Le résultant de ces deux équations, que nous désignerons par $R_{12}$ est le produit

$$
\Pi f_{2}\left(x_{1}, x_{2}\right)
$$

où les $x_{1}, x_{2}$ sont définis par l'égalité (identique par rapport aux $u$ )

$$
\Pi\left(u_{1} x_{1}+u_{2} x_{2}\right)=f_{1}\left(u_{2},-u_{1}\right) \text {. }
$$

Cette expression $R_{12}$ est liée à l'expression analogue $R_{21}$ par la relation

$$
R_{12}=(-\mathrm{I})^{m_{1} m_{2}} R_{21} \text {. }
$$

3. Les fonctions $f_{1}\left(x_{1}, x_{2}\right), f_{3}\left(x_{1}, x_{2}\right)$ étant représentées symboliquement par $a_{x}^{m_{1}}, b_{x}^{m_{2}}$, la quantité invariante $R_{12}$ s'exprimera par une combinaison de déterminants symboliques.

Prenons d'abord $f_{2}$ seul sous forme symbolique, $f_{1}$ étant donné par l'égalité (2). Il faudra remplacer $R_{12}$ par la quantité

$$
\frac{\mathrm{I}}{\sqrt{m_{1}}} \sum\left(b_{1}^{(1)} x_{1}^{(1)}+b_{2}^{(1)} x_{2}^{(1)}\right)^{m_{2}}\left(b_{1}^{(2)} x_{1}^{(2)}+l_{2}^{(2)} x_{2}^{(2)}\right)^{m_{2}} \ldots\left(b_{1}^{\left(m_{1}\right)} x_{1}^{\left(m_{1}\right)}+b_{2}^{\left(m_{2}\right)} x_{2}^{\left(m_{1}\right)}\right)^{m_{2}}
$$

ò la sommation est étendue aux $\mid m_{1}$ permutations des $m_{1}$ couples $\left(b_{1}^{(1)}, b_{2}^{(1)}\right),\left(b_{1}^{(2)}, b_{2}^{(2)}\right), \ldots$ Le problème de l'expression symbolique $\mathrm{du}$ résultant est donc identique au suivant: $L a$ forme

$$
f_{1}\left(u_{2},-u_{1}\right)=\left(u_{1} x_{1}^{(1)}+u_{2} x_{2}^{(1)}\right) \ldots\left(u_{1} x_{1}^{\left(m_{1}\right)}+u_{2} x_{2}^{\left(m_{1}\right)}\right)
$$

étant représentée symboliquement par $(a u)^{m_{1}}$ (ou encore par $u_{a_{1}}^{m_{1}}$, en posant 
$\left.a_{1}=\alpha_{2}, a_{2}=-\alpha_{1}\right)$, trouver sous forme symbolique la somme des puissances $m_{2}^{\text {èmes }}$ des $\underline{m_{1}}$ produits tels que

$$
\bar{\omega}=\left(u_{1}^{(1)} x_{1}^{(1)}+u_{2}^{(1)} x_{2}^{(1)}\right)\left(u_{1}^{(2)} x_{1}^{(2)}+u_{2}^{(2)} x_{2}^{(2)}\right) \ldots\left(u_{1}^{\left(m_{1}\right)} x_{1}^{\left(m_{1}\right)}+u_{2}^{\left(m_{1}\right)} x_{2}^{\left(m_{1}\right)}\right) .
$$

Les deux expressions $\Sigma \overline{\boldsymbol{w}}^{m_{2}}$ et $R_{12}$ sont effectivement équivalentes moyennant le remplacement des $u$ par les $b$.

4. Le produit $\bar{\omega}$ est racine d'une équation de degré $\mid m_{1}$ dont les coefficients sont des contrevariants de $f_{1}$; le dernier terme de cette équation est $d u$ reste immédiatement connu. Il est évidemment

$$
\left[f_{1}\left(u_{2}^{(1)},-u_{1}^{(1)}\right) f_{1}\left(u_{2}^{(2)},-u_{1}^{(2)}\right) \ldots f_{1}\left(u_{2}^{\left(m_{1}\right)},-u_{1}^{\left(m_{1}\right)}\right)\right] \stackrel{\left(m_{1}-1\right)}{=}
$$

Il suffira donc d'avoir formé les $m_{1}-$ I premières sommes de puissances pour pouvoir calculer toutes les autres.

Le nombre $\mid m_{1}-1$ augmentant rapidement avec $m_{1}$, cette remarque n'a pas d'application à partir de $m_{1}=4$. Mais c'est elle que l'on emploie dans le cas de $m_{1}=2$, et elle conduit directement au calcul de $R_{12}$ pour $m_{1}=3$. M. Gordan a en effet ${ }^{\prime}$ formé les résultants de l'équation du $3^{\mathrm{e}}$ degré avec les équations de degré inférieur à 6 . Or ceci suffit, d'après ce qui vient d'être dit, pour passer au cas de $m_{2}$ quelconque.

En désignant par $\frac{S_{1}}{6}, \frac{S_{2}}{6}, \ldots, \frac{S_{5}}{6}$ les expressions de $R_{12}$ pour les cinq premiers degrés, on devra ${ }^{2}$ en déduire les quantités

$$
\begin{gathered}
A_{1}=-S_{1}, \quad A_{2}=\frac{1}{2} S_{1}^{2}-\frac{1}{2} S_{2}, \quad A_{3}=-\frac{S_{1}^{3}}{6}+\frac{S_{1} S_{2}}{2}-\frac{S_{3}}{3}, \\
A_{4}=\frac{S_{1}^{4}}{24}-\frac{S_{1}^{2} S_{2}}{4}+\frac{S_{1} S_{3}}{3}+\frac{S_{2}^{2}}{8}-\frac{S_{t}}{4} \\
A_{5}=-\frac{S_{1}^{5}}{120}+\frac{S_{1}^{3} S_{2}}{12}-\frac{S_{1} S_{2}^{2}}{8}-\frac{S_{1}^{2} S_{3}}{6}+\frac{S_{2} S_{3}}{6}+\frac{S_{1} S_{4}}{4}-\frac{S_{5}}{5}, \\
A_{6}=(a b)^{3}\left(a^{\prime} b^{\prime}\right)^{3}\left(a^{\prime \prime} b^{\prime \prime}\right)^{3}\left(a^{\prime \prime \prime} b^{\prime \prime \prime}\right)^{3}(a b)^{3}\left(a^{\mathrm{IV}} b^{\prime}\right)^{3}\left(a^{\mathrm{V}} b^{\prime \prime}\right)^{3},
\end{gathered}
$$

Math. Annalen, t. 3, pag. 355 et suiv.

2 Voir Serret, Algèbre supérieure, t. I, pag. 460. 
et le résultant de la forme cubique avec une autre de degré quelconque $m_{2}$ sera $^{1}$

$$
\frac{1}{6} \sum \frac{(-1)^{\lambda_{1}+\lambda_{2}+\ldots+\lambda_{6} m_{2} \mid\left(\lambda_{1}+\lambda_{2}+\ldots+\lambda_{6}-1\right)}}{\left|\lambda_{1}\right| \lambda_{2} \ldots \mid \lambda_{6}} A_{1}^{\lambda_{1}} A_{2}^{\lambda_{2}} \ldots A_{6}^{\lambda_{6}},
$$

$\lambda_{1}, \lambda_{2}, \ldots, \lambda_{6}$ désignant successivement les différents systèmes d'exposants entiers et positifs qui vérifient la condition

$$
\lambda_{1}+2 \lambda_{2}+\ldots+6 \lambda_{6}=m_{2}
$$

il faut seulement observer que, dans les multiplications, les $a$ doivent être remplacés chaque fois par des symboles synonymes, mais non les $b$.

5. La résolution $d u$ problème $d u n^{\circ} 3$ permettrait de résoudre sous sa forme générale le problème de l'expression des fonctions symétriques, tel qu'il se pose dans la théorie des formes. En effet, de l'expression $\Sigma \bar{\omega}^{m_{2}}$ peut se déduire par formation polaire l'expression plus générale

$$
P=\Sigma\left(u_{1}^{(1)} x_{1}^{(1)}+u_{2}^{(1)} x_{2}^{(1)}\right)\left(u_{1}^{(2)} x_{1}^{(1)}+u_{2}^{(9)} x_{2}^{(1)}\right) \ldots=\Sigma \Pi_{\gamma_{k}^{i}}
$$

où chaque couple $x_{1}^{(k)}, x_{2}^{(k)}\left(k=1,2, \ldots, m_{1}\right)$ figure dans $m_{2}$ facteurs, les couples des $u$ correspondants étant ou non différents. La sommation est étendue aux $\mid m_{1}$ permutations des $x$ et l'on a posé

$$
r_{k}^{i}=u_{1}^{(i)} x_{1}^{(k)}+u_{2}^{(i)} x_{2}^{(k)}
$$

Or cette expression est la fonction symétrique la plus générale dans les termes de laquelle n'entrent que des facteurs contrevariants. Les facteurs covariants se ramènent d'ailleurs aux précédents par la substitution $u_{1}=y_{2}, u_{2}=-y_{1}$ et les facteurs invariants par l'identité

$$
x_{1}^{(1)} x_{2}^{(2)}-x_{2}^{(1)} x_{1}^{(2)}=\left|\begin{array}{ll}
\frac{\partial}{\partial u_{1}^{(1)}} & \frac{\partial}{\partial u_{2}^{(1)}} \\
\frac{\partial}{\partial u_{1}^{(2)}} & \frac{\partial}{\partial u_{2}^{(2)}}
\end{array}\right| \gamma_{1}^{1} \gamma_{2}^{2} .
$$

6. Le calcul d'une expression $P$ conduit à une quantité du même degré total $m_{1} m_{2}$ par rapport aux lettres $u$ et aux lettres $a$, et qui par

' Serret, ibid., pag. 449 
suite peut s'écrire à l'aide de facteurs du seul type (au) (ou $u_{\alpha}$ ); car si un terme quelconque renferme des facteurs $\left(u u^{\prime}\right)$ il renferme un même nombre de facteurs $\left(\alpha \alpha^{\prime}\right)$ que l'on peut accoupler aux premiers pour utiliser l'identité

$$
\left(\alpha \alpha^{\prime}\right)\left(u u^{\prime}\right)=u_{\alpha} u_{a^{\prime}}^{\prime}-u_{\alpha^{\prime}} u_{\alpha}^{\prime} .
$$

Ce résultat peut d'ailleurs être atteint de plusieurs façons différentes, d'où, pour la quantité cherchée, plusieurs formes symboliques. équivalentes. Néanmoins certaines de ces formes devront être considérées de préférence aux autres.

Remarquons en effet qu'une expression symbolique où n'entrent que des facteurs $u_{a}$ peut immédiatement s'exprimer en fonction des $x$ par la relation

$$
u_{a}^{(1)} u_{\alpha}^{(2)} \ldots u_{\alpha}^{\left(m_{1}\right)}=\frac{\mathrm{I}}{\mid \underline{m_{1}}} \Sigma_{\gamma_{1}^{1}}^{1} \gamma_{2}^{2} \ldots \gamma_{m_{1}}^{m_{1}}
$$

où dans le second membre les indices inférieurs subissent toutes les permutations possibles.

L'expression symbolique devra être telle que cette opération conduise à une quantité $P_{1}$ identique à $P$. Mais deux cas peuvent se présenter. Ou bien l'égalité $P_{1}=P$ est une identité par rapport aux $\gamma$; ou bien elle ne devient telle qu'en remplaçant ces $r$ par leurs valeurs (3). Dans le premier cas nous dirons, pour abréger, que la relation $P_{1}=P$ est essentiellement identique. Nous allons faire voir dans un instant que pour toute quantité $P$ existe une forme symbolique satisfaisant à cette condition, autrement dit une forme symbolique essentiellement équivalente.

7. L'avantage des formes symboliques essentiellement équivalentes est de se conserver lorsqu'on passe à un nombre de variables supérieur à deux.

Soit

$$
u_{a}^{m}=\prod_{k=1}^{m} r_{k}
$$

où $\gamma_{k}$ a cette fois la valeur

$$
r_{k}=u_{1} x_{1}^{(k)}+\ldots+u_{h} x_{h}^{(k)}
$$


une forme, décomposablè en facteurs linéaires, à $h$ variables, $h$ étant un nombre que nous pourrons faire varier à volonté. Formons dans ces nouvelles conditions la quantité

$$
P=\Sigma \pi_{k}^{i}
$$

Cette quantité peut s'exprimer sous une forme symbolique qui, étant du même degré par rapport aux $u$ et aux $\alpha$, peut être ramenée comme précédemment à ne contenir que des facteurs $u_{u k}$. Comme de cette forme symbolique on revient à la forme primitive en employant précisément la mème relation (6) que dans le cas de $h=2$, une forme symbolique essentiellement équivalente trouvée dans ce dernier cas conviendra également à notre problème actuel.

Inversement, si une forme symbolique de l'expression $P$ convient pour $h$ suffisamment grand, elle est essentiellement équivalente.

Ceci revient à dire que l'on peut prendre $h$ assez grand pour que les $\gamma_{i}^{i}$ soient tous indépendants. Or, dans l'hypothèse contraire, comme toute relation existant entre ces quantités pour une certaine valeur de $h$ reste évidemment vraie pour les valeurs moindres, il existerait pour le nombre $h$ une certaine valeur à partir de laquelle le système $E$ des équations qui lient les $\gamma$ entre eux ne changerait plus jusqu'à $h=\infty$ : Ce système devrait être tel que, vérifie pour ccrtaines valeurs $\gamma_{k}^{\prime i}$ des $\gamma$ et pour d'autres valeurs $\gamma_{k}^{\prime \prime}$, il soit par cela même vérifié pour les valeurs $\gamma_{k}^{\prime i}+\gamma_{k}^{\prime \prime i}$ (cette addition revenant au doublement du nombre h). De pareilles équations ne peuvent être que linéaires, ce qui est manifestement impossible dans l'espèce.

En particulier, ceci nous permet d'affirmer l'existence d'une expression symbolique essentiellement équivalente pour toute expression $P$.

8. La formation générale de l'expression $\boldsymbol{P}$ permettrait d'obtenir, pour un nombre quelconque $h$ de variables, les fonctions symétriques invariantes des systèmes $x_{i}^{(k)}, \ldots, x_{h}^{(k)}$ qui figurent dans une forme du type (7).

En effet, de telles fonctions peuvent s'exprimer à l'aide de facteurs symboliques du type $r_{k}^{i}$ et de facteurs déterminants: ces derniers se ramènent aux premiers à l'aide d'une formule analogue à la formule (4). 
9. Si nous appliquons spécialement au résultant de deux formes binaires $(a u)^{m}=u_{o}^{m}$ et $(b u)^{n}=u_{\beta}^{n}$, nous pouvons nous demander s'il existe pour ce résultant une forme symbolique qui, lorsqu'on remplace, soit les lettres $b$, soit les lettres $a$ par des $u$, soit dans les deux cas essentiellement équivalente.

Pour résoudre cette question, nous considérerons les deux formes

$$
F=u_{a}^{m}, \quad \Phi=b_{x}^{n}
$$

à un nombre quelconque $h$ de variables (les variables tangentielles $u$ pour l'une, les variables ponctuelles $x$ pour l'autre).

Si la première forme est décomposable en facteurs linéaires

$$
F=\prod_{i=1}^{m}\left(u_{1} \xi_{1}^{(i)}+u_{2} \xi_{2}^{(i)}+\ldots+u_{h} \xi_{h}^{(i)}\right)
$$

le produit des résultats de substitution des systèmes des $\xi$ dans la deuxième forme s'exprime par un invariant commun à $F$ et $\boldsymbol{\phi}$. Soit $A$ l'expression ainsi obtenue, qui n'est pas complètement déterminée en ce sens qu'on peut lui ajouter une quelconque des expressions $G$ qui s'annulent lorsque $F$ est un produit de facteurs linéaires.

Si de même $\Phi$ se décompose en facteurs du premier degré

$$
\Phi=\prod_{k=1}^{n}\left(g_{1}^{(k)} x_{1}+\ldots+g_{h}^{(k)} x_{h}\right)
$$

le produit des résultats de substitution des différents systèmes de $x$ dans $F$ sera un invariant commun $B$, déterminé seulement aux expressions $D$ près qui s'annulent lorsque $\Phi$ admet une telle réduction.

Je dis que l'on peut disposer des quantités additionnelles $C$ et $D$ de manière à rendre les invariants $A$ et $B$ identiques.

Pour cela, remarquons que lorsque $F$ et $\Phi$ sont tous deux décomposables en facteurs linéaires, on a nécessairement $A=B$, ces deux expressions représentant toutes deux le produit

$$
\prod_{i=1}^{m} \prod_{k=1}^{n}\left(\xi_{1}^{(i)} g_{1}^{(i)}+\ldots+\xi_{h}^{(i)} g_{h}^{(k)}\right)
$$

Il ressort de là presque évidemment que la différence $B$ peut s'exprimer par la somme d'une expression $C$ et d'une expression $D$ : c'est là 
un fait général dont un cas particulier est fourni par le théoréme de Nöтнеn sur les courbes planes passant par l'intersection de deux courbes données. Ici cette conclusion peut s'établir directement de la façon suivante.

Supposons $F$ réductible et représenté par le produit (8). La différence $A-B$, qui est un polynòme homogène et symétrique par rapport aux $\xi$, est par suite une somme de termes de la forme $P Q$, où $P$ est une fonction symétrique élémentaire des $\xi$ et $Q$ une fonction des coefficients de $\Phi$. Cette dernière est nécessairement une expression $D$, puisque la somme $\Sigma P Q$ est nulle, quels que soient les $\xi$, lorsque $F$ et $\Phi$ ont respectivement les formes (8), (9). Si donc nous remplaçons les fonctions symétriques $P$ par leurs valeurs en fonction des coefficients de $F$, nous aurons une expression $D$, identiquement égale à la différence $A-B$ chaque fois que $F$ est réductible et qui par suite n'en différera, pour $F$ quelconque, que d'une expression $C$, ce que nous voulions établir.

Dans l'identité

$$
A-B=C+D
$$

les polynòmes $C$ et $D$ ne sont pas, il est rrai, nécessairement des invariants. Mais ils le deviennent par l'application de la méthode ordinaire (substitution linéaire. la plus générule et application répétée de l'opération désignée dans la théorie des formes par la lettre $\Omega$ ).

Dès lors, si $h$ a été pris suffisamment grand, la quantité

$$
A-C \equiv B+D
$$

fournit, en $\mathrm{y}$ réduisant le nombre des variables à deux, une expression jouissant de la propriété cherchée (les formes binaires données étant $\left.u_{a}^{m}, b_{x}^{n}\right)$

Io. Soient maintenant

$$
\begin{cases}(1) & f_{1}=0 \\ (2) & f_{2}=0 \\ (3) & f_{3}=0\end{cases}
$$

trois équations de degrés $m_{1}, m_{2}, m_{3}$ aux inconnues $x, y$. Les termes de plus haut degré des polynòmes $f_{1}, f_{2}, f_{3}$ nous donnent trois formes 
binaires $f_{1}^{0}, f_{2}^{0}, f_{3}^{0}$, dont nous formerons les résultapts. Nous désignerons par $R_{\alpha \beta}^{0}$ le résultant de $f_{a}^{0}, f_{\beta}^{0}$, et, tout d'abord, nous supposerons ces résultants différents de $o$. Désignons par $x^{(i)}, y^{(i)}$ les solutions communes aux équations $\mathrm{E}_{1}, \mathrm{E}_{2}$ et considérons le produit

$$
P_{3}=\prod_{i} f_{3}\left(x^{(i)}, y^{(i)}\right) \text {. }
$$

Ce produit s'exprime rationnellement à l'aide des coefficients des équations $\mathrm{E}$, mais dans son expression figure un dénominateur lequel n'est autre que $R_{12}^{0^{m_{3}}}$. En effet, dans l'équation finale résultant de l'élimination de $y$ entre $\mathrm{E}_{1}$ et $\mathrm{E}_{2}$, le coefficient de $x^{m_{1} m_{2}}$ est $R_{12}^{0}$; il en est de même de l'équation en $x+\lambda y$, à cause des propriétés invariantes de $R_{12}^{0}$. L'expression $P_{3}$, qui est de degré $m_{3}$ par rapport aux $x, y$ ne peut donc renfermer d'autre dénominateur que ce coefficient élevé à la puissance $m_{3}$. Nous poserons

$$
R_{123}=P_{3} \cdot R_{12}^{m_{3}} \text {. }
$$

En partant des équations $\mathrm{E}_{2}, \mathrm{E}_{3}$, nous formerons les expressions analogues $P_{1}$ et $R_{231}=P_{1} . R_{23}^{0^{m_{1}}}$. Nous nous 'proposons de rechercher la relation entre $P_{1}$ et $P_{3}$ (ou entre $R_{231}$ et $R_{123}$ ).

I I. Considérons à cet effet le produit $\Pi_{1}$ analogue à $P_{1}$, mais où $f_{3}$ est remplacé par $f_{3}-\lambda$. Ce produit s'exprime par un polynóme en $\lambda$ qui, égalé à 0 , exprime que $\lambda=f_{3}\left(x^{(i)}, y^{(i)}\right)$. Le produit des racines de l'équation $I I_{1}=O$. (considérée comme équation en $\lambda$ ) est donc $P_{3}$; le terme indépendant de $\lambda$ est $P_{1}$. Le rapport $\frac{P_{1}}{P_{3}}$ est par suite égal au coefficient de $\lambda^{m_{1} m_{2}}$ dans $\Pi_{1}$, multiplié par $(-1)^{m_{1} m_{2}}$.

Or, si nous effectuons la transformation

$$
x=\frac{1}{x^{\prime}}, \quad y=\frac{y^{\prime}}{x^{\prime}}
$$

qui, aux équations $f_{1}=0, f_{2}=0, f_{3}-\lambda=0$, substitue les équations

$$
\left\{\begin{array}{l}
\text { (I) } \quad F_{1} \equiv x^{\prime m_{1}} f_{1}\left(\frac{\mathrm{I}}{x^{\prime}}, \frac{y^{\prime}}{x^{\prime}}\right)=0, \\
(2) \quad F_{2} \equiv x^{\prime m_{2}} f_{2}\left(\frac{1}{x^{\prime}}, \frac{y^{\prime}}{x^{\prime}}\right)=0, \\
\text { (3) } \quad F_{3} \equiv x^{\prime m_{3}}\left[f_{3}\left(\frac{1}{x^{\prime}}, \frac{y^{\prime}}{x^{\prime}}\right)-\lambda\right]=0
\end{array}\right.
$$


le produit $\Pi_{1}$ sera remplacé par le produit

$$
\Pi_{1}^{\prime}=\Pi_{1} \cdot\left(\Pi x^{\prime}\right)^{m_{1}}=\frac{\Pi_{1}}{(\Pi x)^{m_{1}}}
$$

la multiplication étant étendue aux solutions communes aux deux dernières équations $\left(E^{\prime}\right)$.

Pour $\lambda=\infty$, le produit $\Pi_{1}^{\prime}$ prend une valeur finie et déterminée, car l'équation $\mathrm{E}_{3}^{\prime}$ se réduit à $x^{\prime m_{3}}=0$, de sorte que $I_{1}^{\prime}$ devient égal à $\left(\frac{R_{21}^{0}}{b^{m_{1}}}\right)^{m_{3}}$, en désignant par $b$ le coefficient de $y^{m_{2}}$ dans $f_{2}$. Nous aurons donc à multiplier cette quantité par la puissance $m_{1}{ }^{\text {ème }} \mathrm{du}$ coefficient de $\lambda^{m_{2}}$ dans $\Pi(x)$. Ce dernier s'obtient en formant par l'élimination de $y$ entre $f_{2}=0$ et $f_{3}-\lambda=0$ l'équation en $x$. Pratiquant cette élimination par la méthode des fonctions symétriques en partant de léquation $f_{2}=0$, nous voyons que le coefficient de $x^{m_{2} m_{3}}$ est $\frac{R_{z 3}^{0}}{b^{m_{3}}}$ et le terme indépendant de $x$ un polynôme de degré $m_{2}$ en $\lambda$ avec $(-1)^{m_{2}}$ pour premier coefficient, de sorte que le coefficient de $\lambda^{m_{2}}$ dans $\Pi(x)$ a la valeur $\frac{b^{m_{3}}}{h_{23}^{0}}(-1)^{m_{2}\left(1+m_{3}\right)}$, ce qui donne

$$
\frac{P_{1}}{P_{9}}=\frac{R_{21}^{0^{m_{3}}}}{R_{23}^{0^{m_{1}}}}(-I)^{m_{1} m_{2} m_{3}}
$$

ou

$$
R_{s_{31}}=(-\mathrm{I})^{m_{1} m_{2} m_{3}} R_{213}=R_{133} .
$$

Si nous remarquons que la substitution $\left(\begin{array}{l}123 \\ 231\end{array}\right)$ est alternée, tandis que la substitution $\left(\begin{array}{l}23 \mathrm{I} \\ 2 \mathrm{I} 3\end{array}\right)$ ne l'est pas, nous voyons que notre résultat donne l'énoncé suivant: L'expression (10) ne change pas par une substitution alternée des indices I, 2, 3, au lieu qu'une substitution non alternée la multiplie par $(-\mathrm{I})^{m_{1} m_{2} m_{3}}$.

C'est $\dot{a}$ cette expression entière $R_{123}$, indépendante au signe près, comme on le voit, de l'ordre dans lequel on prend les équations, qu'il convient de donner le nom de résultant des polynômes $f_{1}, f_{2}, f_{3}$. 
1 2. Si nous voulons étudier la même question au point de vue projectif, il faudra considérer les trois équations

$$
\begin{cases}(1) & f_{1}=0 \\ (2) & f_{2}=0 \\ (3) & f_{3}=0\end{cases}
$$

comme homogènes et de degrés respectifs $m_{1}, m_{2}, m_{3}$ par rapport aux variables $x_{1}, x_{2}, x_{3}$ qui correspondent à $x, y$ par les relations $x=\frac{x_{1}}{x_{3}}$, $y=\frac{x_{2}}{x_{3}}$

Pour voir ce qui remplacera le résultant $R_{\alpha \beta}^{\curvearrowleft}$, considérons le résultant des deux formes binaires $a_{x}^{m_{\alpha}}, b_{x}^{m_{\beta}}$, lequel s'exprime par des facteurs déterminants symboliques binaires, et appliquons-lui le principe de translation, c'est à dire changeons chaque facteur déterminant binaire en un déterminant ternaire par l'addition d'une lettre $u$. Nous obtenons ainsi un contrevariant commun des formes $f_{\alpha}, f_{\beta}$, que nous nommerons $R_{\alpha \beta}^{u}$. Ce contrevariant se réduit à $R_{a \beta}^{0}$ pour $u_{1}=u_{2}=0, u_{3}=1$. Il peut d'ailleurs se définir sans que l'on connaisse la forme symbolique du résultant. Il suffit d'appliquer aux deux formes données une substitution linéaire où la nouvelle variable correspondant à $x_{3}$ soit $u_{1} x_{1}+u_{2} x_{2}+u_{3} x_{3}$, de former sur les nouvelles formes le résultant $R_{a \beta}^{0}$ et de diviser par la puissance $m_{a} m_{\beta}$ du déterminant de la substitution.

Egalée à $\circ$, la fonction $R_{12}^{u}$ exprime que la droite $u_{x}=0$ passe par un point $\left(f_{1}=0, f_{2}=0\right)$. Elle est identiquement nulle si ces deux courbes ont une partie commune. Sinon, $x_{1}^{(i)}, x_{2}^{(i)}, x_{3}^{(i)}$ désignant les coordonnées de leurs points communs, on a

$$
R_{12}^{u}=\mu \prod_{i}\left(u_{1} x_{1}^{(i)}+u_{2} x_{2}^{(i)}+u_{3} x_{3}^{(i)}\right)
$$

chaque facteur étant élevé à une puissance convenable, qui n'est autre que le degré de multiplicité du point correspondant; et $\mu$ étant un facteur constant. Autrement dit, le rapport

$$
\mu=\frac{k_{12}^{u}}{\prod_{i}\left(u_{1} x_{1}^{(i)}+u_{2} x_{2}^{(i)}+u_{3} x_{3}^{(i)}\right)}
$$


est indépendant des $u$. Mais ce rapport dépend des déterminations adoptées pour les $x$ (lesquels ne sont définis qu'à un facteur de proportionnalité près). Pour $x_{3}^{(1)}=x_{3}^{(2)}=\ldots=1$, on a

$$
\mu=R_{12}^{0} \text {, }
$$

comme on le voit en faisant $u_{1}=u_{2}=0, u_{3}=$ I. Dès lors $R_{123}$ peut être défini: le produit $\prod_{i} f_{3}\left(x_{1}^{(i)}, x_{2}^{(i)}, x_{3}^{(i)}\right)$ multiplié par la puissance $m_{3}^{\text {eme }}$ de la quantité (I3), soit

$$
R_{123}=\prod_{i} f_{3}\left(x_{1}^{(i)}, x_{2}^{(i)}, x_{3}^{(i)}\right)\left[\frac{R_{12}^{u}}{\prod_{i}\left(u_{1} x_{1}^{(i)}+u_{2} x_{2}^{(i)}+u_{3} x_{3}^{(i)}\right)}\right]^{m_{3}}
$$

oi le second membre est cette fois indépendant des $u$ d'une part, des facteurs de proportionnalité qui figurent dans les $x$, de l'autre. En prenant le cas de $\mu=\mathrm{I}$, on voit que le résultant est donné par le produit $\prod_{i} f_{3}\left(x_{1}^{(i)}, x_{2}^{(i)}, x_{3}^{(i)}\right)$, où les $x$ sont définis par l'égalité (identique par rapport $\operatorname{aux} u)$

$$
\prod_{i}\left(u_{1} x_{1}^{(i)}+u_{2} x_{2}^{(i)}+u_{3} x_{3}^{(i)}\right)=R_{12}^{u} .
$$

13. Le résultant considéré sous forme symbolique est donc une expression de la forme $P$ considérée au $n^{\circ} 7$. En particulier on saurait calculer cette expression symbolique si l'on savait résoudre le problème posé au $\mathrm{n}^{\circ}$ 6: trouver pour le résultant de deux formes binaires une expression essentiellement équivalente. Ceci permettrait en effet d'abord de calculer la forme $R_{12}^{u}$, puis de passer au résultant $R_{123}$ par la considération du résultant de deux formes, l'une de degré $m_{1} m_{2}$, l'autre le degré $m_{3}$.

14. L'équation $R_{123}=$ o exprime la condition nécessaire et suffisante pour que les équations $\mathrm{E}$ aient une solution commune (autre que $x_{1}=x_{2}=x_{3}=0$ ). $\mathrm{Si}$ en effet $R_{12}^{u}$ n'est pas identiquement nul, l'équation de définition (1 4) montre que les conditions $R_{123}=$ o, $\prod_{i} f_{3}\left(x_{1}^{(i)}, x_{2}^{(i)}, x_{3}^{(i)}\right)=$ o sont équivalentes. Dans le cas contraire, le résultant, que l'on peut regarder comme composé à l'aide des coefficients de $R_{12}^{u}$ et de $f_{2}$, se réduit à 0 , et d'autre part, il est manifeste que les équations $E$ ont une solution, puisque les deux courbes $f_{1}=0, f_{2}=0$ ont une partie commune, laquelle coupe $f_{3}=o$ en un certain nombre de points. 
15. Le résultant fourni par la méthode dialytique, telle qu'elle est indiquée par $\mathrm{C}_{A Y L E Y}{ }^{1}$ coïncide, au signe près, avec le résultant $R_{123}$. C'est ce que l'on voit en constatant: $I^{\circ}$ que l'expression $R$ de CayLeY est de degré $m_{2} m_{3}$ par rapport aux coefficients de $f_{1}$, d'où résulte que le rapport $\frac{R}{R_{123}}$ est indépendant de $f_{1}$, et pareillement de $f_{2}, f_{3} ; 2^{\circ}$ que les deux expressions $R, R_{123}$ se réduisent toutes deux à I pour $f_{1}=x_{1}^{m_{1}}$, $f_{2}=x_{2}^{m_{2}}, f_{3}=x_{3}^{m_{3}}$.

16. Les conclusions auxquelles nous venons de parvenir s'étendent d'elles-mêmes au cas d'un nombre quelconque $n$ d'inconnues. On peut, à cet égard, répéter identiquement les raisonnements précédents; cette fois, nous les présenterons sous une forme plus simple à certains égards en partant directement des équations rendues hornogènes.

Soient $n+$ I polynómes $f_{1}, f_{2}, \ldots, f_{n+1}$ de degrés $m_{1}, m_{2}, \ldots, m_{n+1}$ respectivement par rapport aux variables $x_{1}, x_{2}, \ldots, x_{n+1}$. Nous allons définir le résultant $R_{1,2, \ldots, n+1}$ de ces polynômes et démontrer que $x, \beta, \gamma, \ldots$ étant une permutation quelconque des indices $1,2, \ldots, n+1$, on a

$$
R_{\alpha \beta \gamma \ldots}=R_{1,2, \ldots, n+1}(-1)^{s m_{1} m_{2} \ldots m_{n+1}}
$$

où $s$ est égal à o ou à I suivant que la substitution $\left(\begin{array}{l}\mathrm{I}, 2, \ldots, n+\mathrm{I} \\ \alpha, \beta, \gamma, \ldots\end{array}\right)$ est ou non alternée.

Supposons pour cela que l'on ait défini le résultant de $n$ équations homogènes à $n$ inconnues. Ce sera un polynòme entier par rapport aux coefficients des différentes équations et dont le degré par rapport aux coefficients de chacune sera marqué par le produit des ordres de toutes les autres. Ce sera un invariant commun à tous les premiers membres. Egalé à zéro, il exprimera la condition nécessaire et suffisante pour que les équations aient une solution commune (non nulle). Enfin il ne changera pas si l'on change l'ordre des équations données, sauf dans le cas ou ces équations sont toutes d'ordre impair et la permutation effectuée non alternée, auquel cas il changera simplement de signe. 
Revenant alors aux équations

$$
\left\{\begin{array}{cc}
(1) & f_{1}=0 \\
(2) & f_{2}=0 \\
\hdashline \cdots & \therefore \cdots \\
(n+1) & f_{n+1}=0
\end{array}\right.
$$

nous considerons d'abord les $n$ premières d'entre elles. En y faisant $x_{n+1}=o$, nous aurons $n$ équations à $n$ inconnues dont nous pourrons former le résultant $R_{1,2, \ldots, n}^{0}$. De cette expression, nous déduirons, comme il a été expliqué plus haut, la forme $R_{1,2, \ldots, n}^{u}$. Egalée à zéro, cette forme exprime que l'équation linéaire $u_{1} x_{1}+\ldots+u_{n+1} x_{n+1}=0$ est vérifiée par une solution commune $x_{1}^{(i)}, x_{2}^{(i)}, \ldots, x_{n+1}^{(i)}$ des $n$ premières équations E. Elle peut ètre identiquement nulle: ceci arrivera alors et alors seulement, que les surfaces représentées par ces $n$ équations auront en commun, non pas seulement un certain nombre de points, mais une multiplicité algébrique $\dot{a}$ au moins une dimension. Sinon, elle sera un produit de facteurs

$$
R_{1,2, \ldots, n}^{u}=\mu \prod_{i}\left(u_{1} x_{1}^{(i)}+\ldots+u_{n+1} x_{n+1}^{(i)}\right)
$$

chaque facteur sous le signe $\Pi$ étant compté un nombre de fois égal au degré de multiplicité de la solution commune correspondante, et $\mu$ étant un nombre indépendant des $u$, mais dépendant du facteur de proportionnalité qui figure dans les $x_{i}$.

Le degré de la forme $R^{u}$ par rapport aux $u$ étant $m_{1} m_{2} \ldots m_{n}$, il est par cela même démontré que ce nombre est celui des solutions communes aux équations $\mathrm{E}_{1}, \mathrm{E}_{2}, \ldots, \mathrm{E}_{n}$.

Cela posé, formons le produit

$$
\begin{gathered}
R_{1,2, \ldots, n+1}=\left(\frac{R_{1,2, \ldots, n}^{u}}{\prod_{i}\left(u_{1} x_{1}^{(i)}+\ldots+u_{n+1} x_{n+1}^{(i)}\right)}\right)^{m_{n+1}} P_{n+1}, \\
P_{n+1}=\prod_{i} f_{n+1}\left(x_{1}^{i j}, \ldots, x_{n+1}^{(i)}\right) .
\end{gathered}
$$

Ce produit est indépendant des $u$, ainsi que des déterminations choisies pour les $x^{(i)}$. On peut en particulier choisir ceux-ci de manière que $\mu=\mathrm{I}$ et définir notre quantité commé le produit

$$
R_{1,2, \ldots, n+1}=\prod_{i} f_{n+1}\left(x_{1}^{(i)}, \ldots, x_{n+1}^{(i)}\right)
$$


les quantités $x$ étant définies par l'identité

$$
\prod_{i}\left(u_{1} x_{1}^{(i)}+\ldots+u_{n+1} x_{n+1}^{(i)}\right)=R_{1,2, \ldots, n}^{u} .
$$

L'expression $\dot{R}_{1,2, \ldots, n+1}^{u}$ est donc une fonction entière des coefficients de $R_{1,2 \ldots, n}^{u}$ et des coefficients de $f_{n+1}$, de degré $m_{n+1}$ par rapport aux premiers, de degré $m_{1} m_{2} \ldots m_{n}$ par rapport aux seconds; par suite une fonction entière des coefficients des équations $\mathrm{E}$, et dont le degré par rapport aux coefficients de chaque équation est marqué par le produit des ordres des autres. C'est un invariant commun aux polynómes $f_{1}, \ldots, f_{n+1}$, car dans l'expression (i6) tous les facteurs sont invariants. Son expression symbolique comprend $m_{1} m_{2} \ldots m_{n+1}$ facteurs déterminants symboliques. On saurait d'ailleurs former cette expression symbolique si l'on savait trouver pour le résultant de deux formes binaires une expression essentiellement équivalente.

I 7. $R_{1,2, \ldots, n+1}=$ o est la condition nécessaire et suffisante pour que les équations E aient une solution commune non nulle. C'est ce qui résulte immédiatement de la formule (16) si $R_{1,2, \ldots, n}^{u}$ n'est pas identiquement nul. Si au contraire on a $R_{1,2, \ldots, n}^{u} \equiv 0$, on aura aussi $R_{1,2, \ldots, n+1}=0$, puisque $R_{1,2, \ldots, n+1}$ est une fonction homogène des coefficients de $R_{1,2, \ldots, n}^{u}$. D'ailleurs les équations $\mathrm{E}$ auront une solution commune, car les $n$ premières d'entre elles définissent une multiplicité algébrique à au moins une dimension, laquelle coupe nécessairement la surface $f_{n+1}=0$.

Il est donc légitime de nommer résultant cette expression $R_{1,2, \ldots, n+1}$.

I 8. Soient maintenant $\alpha, \beta, \gamma, \ldots, \eta, \theta$ les indices $\mathrm{I}, 2,3, \ldots, n+\mathrm{I}$ rangés dans un ordre différent et formons l'expression, analogue a $R_{1,2,3, \ldots, n+1}$

$$
\begin{aligned}
R_{\alpha, \beta, \gamma, \ldots, \gamma_{,} \theta} & =\left[\frac{R_{u, \beta, \gamma}^{u} \ldots, \eta}{\prod_{k}\left(u_{1} x_{1}^{(k)}+\ldots+u_{n+1} x_{n+1}^{(k)}\right)}\right]^{m_{\theta}} P_{\theta}, \\
P_{\theta} & =\prod_{k} f_{\theta}\left(x_{1}^{(k)}, x_{2}^{(k)}, \ldots, x_{n+1}^{(k)}\right) .
\end{aligned}
$$

Le rapport $\frac{R_{a, \beta, \gamma, \ldots, \theta}}{R_{1,2,3, \ldots, n+1}}$ est indépendant des coefficients de l'une quelconque, $f_{1}$ par exemple, des équations $\mathrm{E}$. En général, en effet, les équations $\mathrm{E}_{2}, \mathrm{E}_{3}, \ldots, \mathrm{E}_{n+1}$ ont leurs $m_{2} m_{3} \ldots m_{n+1}$ solutions communes distinctes 
(puisqu'en y faisant $x_{n+1}=\mathrm{I}$, de manière à les prendre sous forme non homogène, $I^{\circ}$ il n'y aura pas, en général, de solutions commune infinie, le résultant que nous avons appelé $R_{2,3, \ldots, n+1}^{0}$ n'étant pas identiquement nul; $2^{\circ}$ le résultant des équations $\mathrm{E}_{2}, \mathrm{E}_{3}, \ldots, \mathrm{E}_{n+1}$ et de leur déterminant fonctionnel, qui est une fonction entière des coefficients de ces équations, n'est pas identiquement nul, ainsi qu'on le voit en prenant pour ces équations des produits de facteurs linéaires quelconques). Considérées comme équations entre les coefficients de $f_{1}$, les équations $R_{\alpha, \beta, \ldots, \phi}=0, R_{1,2, \ldots, n+1}=0$ représentent donc la même multiplicité algébrique, 'en général sans partie multiple; elles coïncident done bien à un facteur près indépendant de $f_{1}$. Cette conclusion, établie dans le cas où les $n$ dernières équations $\mathrm{E}$ ont leurs solutions communes distinctes, subsiste par cela même d'une façon absolument générale.

On peut dès lors, dans l'évaluation du rapport $\frac{R_{\alpha, 3,3, \ldots, \theta}}{h_{1,2, \ldots, n+1}}$, supposer les polynômes $f_{1}, f_{2}, \ldots, f_{n+1}$ remplacés respectivement par les puissances $m_{1}^{\text {ème }}, m_{2}^{\text {ème }}, \ldots, m_{n+1}$ ème d'autant de polynômes du premier degré.

D'autre part, le résultant $R_{a, \beta_{3}, \ldots, \ldots, \theta}$ des formes $f_{1}^{p}, f_{2}, \ldots, f_{n+1}$ est la puissance $p^{\text {ème }}$ du résultant $R_{\alpha, 3,3, \ldots, \theta}$ relatif aux formes $f_{1}, f_{2}, \ldots, f_{n+1}$. Cela est évident si $\theta=\mathrm{I}$. Dans le cas contraire, on voit immédiatement d'après la formule (16') qu'il suffit d'établir la propriété en question pour la quantité $R_{u, \hat{\beta}, \gamma, \ldots, \gamma}^{u}$. Or celle.ci, se déduisant du résultant de $n$ équations homogènes à $n$ inconnues ne change pas par une substitution alternée, substitution par laquelle nous pouvons rendre $\eta$ égal à I.

Dès lors le rapport $\frac{R_{a, 3, \ldots, \ldots, \theta}}{h_{1,2,3, \ldots, n+1}}$ est là puissance $m_{1} m_{2} \ldots m_{n+1}$ ème de ce qu'il serait si les polynômes donnés étaient du premier degré. Mais, dans ce cas, le résultant, qui se réduit au déterminant des $(n+1)^{2}$ coefficients, est une fonction alternée par rapport aux indices $\alpha, \beta, \gamma, \ldots, \theta$. Nous pouvons done écrire

$$
\boldsymbol{R}_{\alpha_{,}, \xi \gamma, \ldots, \theta}=(-1)^{s m_{1} m_{2} \ldots m_{n+1}} R_{1,2,3, \ldots, n+1}
$$

et nous aurons ainsi démontré toutes les propriétés énoncées.

19. Les formules (16) et (17) permettent de comparer entre eux les produits $P_{1}, P_{2}, \ldots, P_{n+1}$. Que devient cette comparaison lorsque 
nos équations ont des solutions communes? Prenons, pour simplifier, le cas de trois équations $f_{1}=0, f_{2}=0, f_{3}=0$ représentant trois courbes qui ont un certain nombre de points communs $A$. Sur un cycle de $f_{1}$ ayant pour origine un de ces points, $f_{2}$ sera de l'ordre $l$ et $f_{3}$ de l'ordre $l^{\prime}$, de sorte que le nombre des intersections $B$ de $f_{1}, f_{2}$ non communes avec $f_{3}$ sera $m_{1} m_{1}-\Sigma l$ et le nombre des intersections $C$ de $f_{1}$ et de $f_{3}$, non communes avec $f_{2}, m_{1} m_{3}-\Sigma l$. Proposons-nous de comparer le produit des valeurs de $f_{3}$ aux points $B$ avec le produit des valeurs de $f_{2}$ aux points $C$.

Remplaçons, dans ce but, $f_{3}$ par $f_{3}+\lambda \varphi_{3}\left(\varphi_{3}\right.$ étant un polynôme quelconque de degré $m_{3}$ ); nous voyons que le produit des valeurs de $f_{3}$ aux points $B$, multiplié par le produit des valeurs de $\varphi_{3}$ aux points $A$, égale le coefficient de $\lambda^{\Sigma l}$ dans le produit $P_{3}$ relatif aux polynômes $f_{1}, f_{2}, f_{3}+\lambda \varphi_{3}$, lequel produit est égal, aux facteurs près dont nous avons donné l'expression plus haut, au produit des valeurs de $f_{2}$ aux points $f_{1}=0, f_{3}+\lambda \varphi_{3}=0$. Ces derniers peuvent se diviser en deux catégories: $m_{1} m_{3}-\Sigma l^{\prime}$ qui, pour $\lambda$ infiniment petit, tendent vers les points $C$ et $\Sigma l^{\prime}$ qui tendent vers les points $A$.

Sur chaque cycle de $f_{1}$ ayant pour origine un point $A,{ }^{1}$ donnonsnous les développements de $f_{2}, f_{3}, \varphi_{3}$ :

$$
\begin{aligned}
f_{2} & =t^{l}(a+b t+\ldots), \\
f_{3} & =t^{\prime}\left(a^{\prime}+b^{\prime} t+\ldots\right), \\
\varphi_{3} & =\alpha+\beta t+\ldots
\end{aligned}
$$

Les $l^{\prime}$ valeurs de $t$ correspondant aux points d'intersection de $f_{1}$ avec $f_{3}+\lambda \varphi_{3}$ auront leurs parties principales données par $a^{\prime} t^{l^{\prime}}+\lambda \alpha=0$, de sorte que leur produit aura pour valeur principale $(-1)^{r^{\prime}} \frac{\lambda \alpha}{a^{\prime}}$ et le produit des valeurs de $f_{2},(-1)^{l l^{\prime}} \lambda^{l} \alpha^{l} \frac{a^{l^{\prime}}}{a^{\prime l}}$. Si nous supprimons le facteur $\lambda^{l}$ et remarquons que les facteurs $\alpha^{l}$ donnent le produit des valeurs de

1 Il est bien entendu que les dévoloppements de $x_{1}, x_{2}, x_{3}$ sur le cycle doivent être tels que, pour $t=0$, on trouve les valeurs mêmes qui figurent dans les formules (13) et suivantes (et non simplement des valeurs proportionnelles).

Aeta mathematica. 20. Imprimê le 14 septembre 1896. 
$\varphi_{3}$ aux points $A$, nous voyons que le cycle en question figurera par le facteur (- I $)^{n l^{\prime}} \frac{a^{l^{\prime}}}{a^{a^{\prime}}}$, c'est à dire, au signe près, la vraie valeur de $\frac{f_{2}^{r^{\prime}}}{f_{3}^{l}}$ sur le cycle en question. ${ }^{1}$ Tel est donc le facteur qu'il faudra, dans la formule de comparaison, introduire pour chaque intersection commune que l'on supprimera.

20. En particulier, ceci nous permettra de voir ce que devient la formule

$$
R=\prod_{i} f_{3}\left(x^{(i)}, y^{(i)}\right) R_{12}^{0^{m_{3}}}
$$

qui exprime le résultant en supposant connus les points d'intersection de $f_{1}=\mathrm{o}$ et de $f_{2}=0$, lorsqu'un ou plusieurs de ces points sont à l'infini.

Soient

$$
x^{(i)}, y^{(i)}, \mathrm{I} \quad\left(i=1,2, \ldots, m_{1} m_{2}-\mu\right)
$$

les points communs de $f_{1}, f_{2}$ situés à distance finie,

$$
a^{(k)}, b^{(k)}, \mathrm{O} \quad(k=1,2, \ldots, \mu)
$$

les points communs à l'infini. On a

$$
\left(\mathrm{I} 4^{\prime}\right) \quad R=\prod_{(i)} f_{3}\left(x^{(i)}, y^{(i)}\right) \prod_{k} f_{3}^{0}\left(a^{(k)}, b^{(k)}\right)\left[\frac{R_{12}^{u}}{\prod_{i}\left(u_{1} x^{(i)}+u_{2} y^{(i)}+u_{3}\right) \prod_{k}\left(u_{1} a^{(i)}+u_{2} b^{(k)}\right)}\right]^{m_{3}},
$$

et c'est la quantité $\frac{R_{12}^{u}}{\prod_{i}^{u}\left(u_{1} x^{(i)}+u_{2} y^{(i)}+u_{3}\right) \prod_{k}\left(u_{1} e^{(k)}+u_{2} b^{(k)}\right)}$ qu'il nous reste à évaluer. Or $R_{12}^{u}$ est le résultant des polynòmes $f_{1}, f_{2}, u_{1} x_{1}+u_{2} x_{3}+u_{3} x_{3}$ Autrement dit, si

$$
\xi_{1}^{(h)}, \xi_{2}^{(h)}, \xi_{3}^{(h)} \quad\left(h=1,2, \ldots, m_{1}\right)
$$

désignent les points d'intersection de $f_{1}=0, u_{1} x_{1}+u_{2} x_{2}+u_{3} x_{3}=0$, on aura

(18) $R_{12}^{u}=\prod_{h} f_{2}\left(\xi_{1}^{(h)}, \xi_{2}^{(h)}, \xi_{3}^{(h)}\right)\left[\frac{f_{1}\left(u_{2} v_{3}-v_{2} u_{3}, u_{3} v_{1}-u_{1} v_{3}, u_{1} v_{2}-v_{1} u_{2}\right)}{\prod_{h}\left(v_{1} \xi_{1}^{(h)}+v_{2} \xi_{2}^{(h)}+v_{3} \xi_{3}^{(h)}\right)}\right]^{m_{2}}$.

I D'après la note précédente, il est clair que cette vraie valeur dépendra, comme cela doit être, du facteur de proportionnalité qui figure dans les coordonnées de l'origine du cycle. 
Divisant cette valeur par le dénominateur

$$
\prod_{i}\left(u_{1} x^{(i)}+u_{2} y^{(i)}+u_{3}\right) \prod_{k}\left(u_{1} a^{(k)}+u_{2} b^{(k)}\right),
$$

nous allons pouvoir prendre pour la droite $u_{1} x_{1}+u_{2} x_{2}+u_{3} x_{3}=$ o la droite de l'infini $u_{1}=u_{2}=0, u_{3}=\mathrm{I}$. Dans ces conditions, en effet, les points $\xi_{1}^{(h)}, \xi_{2}^{(h)}, \xi_{3}^{(h)}$ sont, d'une part, ${ }^{1}$ les points $a^{(k)}, b^{(k)}$, o, d'autre part, les autres points à l'infini $\left(a^{\left(k^{\prime}\right)}, b^{\left(k^{\prime}\right)}\right.$, o), points dont nous pouvons supposer les coordonnées choisies de manière $\grave{a}$ ce que le produit

$$
\prod_{k^{\prime}}\left(v_{1} a^{\left(k^{\prime}\right)}+v_{2} b^{\left(k^{\prime}\right)}\right)=f_{1}^{\prime 0}\left(v_{2},-v_{1}\right)
$$

soit égal au quotient de $f^{0}\left(v_{2},-v_{1}\right) \operatorname{par}^{2} \Pi\left(v_{1} a^{(k)}+v_{1} b^{(k)}\right)$. Dans ces conditions, le second facteur de la valeur (18) de $R_{12}^{u}$ devient égal à l'unité. Quant au rapport $\frac{\prod_{h} f_{2}\left(\xi_{1}^{(h)}, \xi_{2}^{(h)}, \xi_{3}^{(h)}\right)}{\prod_{i}\left(u_{1} x^{(i)}+u_{2} y^{(i)}+u_{3}\right) \prod_{k}\left(u_{1} a^{(k)}+u_{2} b^{(k)}\right)}$, il se présente sous la forme $\frac{o}{o}$, mais nous venons d'apprendre dans le numéro précédent à lever cette indétermination, et nous obtenons

$$
R=\prod_{i} f_{3}\left(x^{(i)}, y^{(i)}\right) \prod_{k} f_{3}^{0}\left(a^{(k)}, b^{(k)}\right)\left(\Pi f_{2}^{0}\left(a^{\left(k^{\prime}\right)}, b^{\left(k^{\prime}\right)}\right) \Pi L\right)^{m_{3}}
$$

Les facteurs $L$ sont relatifs aux différentes branches infinies de $f_{1}$. Si sur une telle branche, $f_{2}$ est d'ordre $l, x_{3}$ d'ordre $l$ ', le facteur $L$ sera la vraie valeur de $\frac{f_{2}^{l^{\prime}}}{x_{3}^{l}}$, multipliée par $(-\mathrm{I})^{l^{\prime}}$.

Le facteur $\Pi f_{2}^{0}\left(a^{\left(k^{\prime}\right)}, b^{\left(k^{\prime}\right)}\right)$ se calcule sans que l'on connaisse les quantités $a^{\left(k^{\prime}\right)}, b^{\left(k^{\prime}\right)}$. C'est le résultant des formes binaires $f_{1}^{\prime 0}, f_{2}^{0}$.

1 Ces points ne sont pas, il est vrai, identiques avec les points $a^{(k)}, b^{(k)}$, o considérés tout à l'heure: ils en diffèrent par les ordres de multiplicité. Les points $a^{(k)}, b^{(k)}$ sont comptés dans la fornule (I $4^{\prime}$ ) avec l'ordre de multiplicité qu'ils ont comme points communs à $f_{1}, f_{2}$; ici on doit leur attribuer la multiplicité avec laquelle ils figurent comme points à l'infini de $f_{1}$.

2 Il est bien entendu que le produit $\prod_{k}\left(v_{1} a^{(k)}+v_{2} b^{(k)}\right)$ n'est pas celui qu, on déduirait du produit $\prod_{k}\left(u_{1} a^{(k)}+u_{2} b^{(k)}\right)$ qui figure dans (I4) en changent les $u$ en $v$, ainsi qu'il résulte de la note précédente. 
21. Ces résultats s'étendent immédiatement à un nombre quelconque d'équations. Le róle que jouait tout à l'heure la courbe $f_{1}=0$ sera joué par la courbe représentée, dans l'espace à $n$ dimensions, par les $(n-\mathrm{I})$ premières équations données.

22. Les conclusions du $n^{\circ}$ I 9 fournissent une identité qu'il est d'ailleurs aisé de vérifier directement, et qui peut être utile dans certains raisonnements. Soient encore les trois courbes $f_{1}=0, f_{2}=0, f_{3}=0$ ayant en commun un point $x_{0}, y_{0}$. Sur un cycle de $f_{1}=0$ en ce point, $f_{2}$ sera de l'ordre $k, f_{3}$ de l'ordre $l$, et le rapport $\frac{f_{2}^{l}}{f_{3}^{k}}$ aura une certaine limite $L$. De même, sur un cycle de $f_{2}$ au mème point, le rapport $\frac{f_{2}^{l^{\prime}}}{f_{1}^{k^{\prime}}}$ (en désignant par $k^{\prime}$ et $l^{\prime}$ les ordres de $f_{3}$ et de $f_{1}$ sur ce cycle) aura une certaine limite $L^{\prime}$, et, sur un cycle de $f_{3}$, le rapport $\frac{f_{1}^{l^{\prime \prime}}}{f_{a}^{k^{\prime \prime}}}$ (où $k^{\prime \prime}, l^{\prime \prime}$ sont les ordres de $\left.f_{1}, f_{2}\right)$ aura une limite $L^{\prime \prime}$. Il résulte évidemment du $n^{\circ} \mathbf{I} 9$ que le produit des quantités $L$ par les quantités $L^{\prime}$ et par les quantités $L^{\prime \prime}$ est égal à $(-\mathrm{I})^{\Sigma k t+\Sigma k^{\prime} t^{\prime}+2 k^{\prime \prime} t^{\prime \prime}}$, du moins si le point $x_{0}, y_{0}$ est le seul point commun; mais on peut toujours supposer qu'il en est ainsi, en ajoutant aux premiers membres des équations de ces courbes des termes de degré assez élevés en $x-x_{0}, y-y_{0}$ pour ne pas changer les quantités $L, L^{\prime}, L^{\prime \prime}$.

\section{II.}

23. Nous avons ćtudié le résultant de plusieurs équations, c'est à dire une certaine fonction symétrique des solutions communes à ces équations. De pareilles quantités, susceptibles de recevoir des applications géométriques plus ou moins simples, ont été considérées par différents auteurs, auxquels elles ont fourni une série de théorèmes de géométric.

C'est ainsi que LaGuerre ${ }^{1}$ a énoncé des théorèmes sur le produit

${ }^{1}$ Comptes Rendus de l'Académie des Sciences, tome 60, pag. 7 I-73; I865. - Bulletin de la Société Philomatique, p. I40; I870. 
des normales menées d'un point à une courbe, sur le produit des distances d'un point aux intersections d'une courbe et d'un cercle, etc.

Un certain nombre de ces théorèmes et d'autres analogues ont été repris par M. Eluing Hoist. ${ }^{1}$

Les fonctions symétriques qui font l'objet de ces différentes propositions appartiennent à une catégorie particulière: il s'agit toujours du produit des, valeurs que prend une fonction rationnelle déterminée aux différents points communs à deux courbes données. Au contraire, d'autres résultats du même genre sont relatifs à l'évaluation, non d'un produit, mais d'une somme de fonctions rationnelles. Tel est, par exemple, le théorème de $\mathrm{J}_{\mathrm{ACOBI}}$ sur la quantité $\sum \frac{U}{D(f, \varphi)}$ (où $D(f, \varphi)$ est le déterminant fonctionnel des polynômes $f, \varphi$ ).

Ces différentes recherches ont été reprises et complétées par M. HumBER'T dans une série d'importants mémoires insérés principalement au Journal de Mathématiques pures et appliquées. ${ }^{2}$ Plus généralement, M. Humber't calcule, à l'aide de la théorie des fonctions fuchsiennes, les intégrales qui interviennent dans le théorème d'ABEL et dont l'expression permet d'obtenir le produit ou la somme de fonctions rationnelles, étendus aux points d'intersection de deux courbes, ou d'une courbe et d'une surface.

Je me propose de faire voir que tous les résultats que nous venons d'énumérer peuvent être considérés comme dérivant des propriétés du résultant.

24. Lorsque la fonction symétrique à évaluer affecte la forme d'un produit, nous sommes immédiatement ramenés au calcul d'un résultant. Le produit

$$
\prod_{i} \frac{F\left(x^{(i)}, y^{(i)}\right)}{\Phi\left(x^{(i)}, y^{(i)}\right)}
$$

\footnotetext{
1 Math. Annalen tome II, pag. 34I-346; 1877. - Bull. de Ia Soc. Math. de France, tome 8, pag. 52-59; 1879 .

${ }^{1}$ Sur le théorème d'Abel et quelques unes de ses applications géométriques, Jour $\mathbf{n}$ al de Math. pures et appliquées, 4 érie, tome 3, pag. 327-405; tome 5, pag. 8 I-I34; tome 6, pag. 233-292. - Sur les courbes cycliques de direction, ibid. tome 5, pag. I 29. Propriétés des arcs des courbes algébriques planes ou gauches, ibid. $5^{\mathrm{e}}$ série, tome I, pag. I 81 ; ete, etc. - Les théorèmes de M. Humbers ont été démontrés par une autre voie dans le Traité des fonctions algébriques et de leurs intégrales de MM. APPELL et Goursat.
} 
étendu aux points d'intersection des courbes $f, \varphi$ est, à un facteur près dépendant des termes du plus haut degré des polynòmes $f, \varphi, F$, $\Phi$, le quotient des résultants des polynômes $f, \varphi, F$ d'une part, $f, \varphi, \phi$ de l'autre; et les autres formes que nous connaissons pour ces résultants nous donneront autant de transformations du produit en question.

Soit, par exemple, le produit

$$
\Pi \psi(x, y)
$$

des valeurs prises par le polynôme entier $\psi(x, y)$ aux points d'intersection des courbes $f=0, \varphi+P \psi=0$. On est conduit à exprimer ce produit par le produit

$$
\Pi(\varphi+P \phi)
$$

étendu aux points d'intersection des courbes $f, \phi$, lequel est indépendant du polynôme entier $P$.

Le rapport des deux produits (2O), (2 I) si le résultant des termes du plus haut degré des polynômes $f, \varphi+P \phi$ n'en dépend pas, ce qui arrivera lorsque $P \phi$ sera de degré inférieur à $\varphi$.

Lorsque la courbe $\psi=0$ est un cercle, cette remarque prend la forme suivante: $S i$ une courbe fixe $f=0$ est coupée par une autre variable, mais dont les points at l'infini et les points d'intersection avec un cercle $\phi=0$ sont fixes, le produit des puissances, par rapport au cercle $\phi$, de la courbe $f=0$ et de la courbe variable est constant.

25. C'est encore dans le même ordre d'idées que rentre le théorème de Laguerre: Si par un point $O\left(x_{0}, y_{0}\right)$ pris dans le plan d'une courbe $f=\mathrm{O}$, on mène un cercle quelconque, le produit des distances du point $O$ aux points d'intersection ${ }^{1}$ est $(2 R)^{n} f_{0}$.

On a en effet à évaluer dans ce cas le produit

$$
\left.P=I I\left(x-x_{0}\right)^{2}+\left(y-y_{0}\right)^{2}\right]=(2 R)^{2 m} I I\left[\left(x-x_{0}\right) \sin \theta-\left(y-y_{0}\right) \cos \theta\right]
$$

1 Il y a iei un léger désaccord avee le résultat donné par LAGUERRE; cela tient à ce que nous supposons la quantité (22) (voir ci-dessous) égale à 1 et non à $2^{m}$, ce qui était la supposition de Laguerre. La forme que nous adoptons ainsi pour $f$ est la forme »normale de M. Elling Holst (loc. cit.). 
étendu aux points d'intersection des courbes $f=0$,

$$
\left(x-x_{0}\right)^{2}+\left(y-y_{0}\right)^{2}-2 R\left(\left(x-x_{0}\right) \sin \theta-\left(y-y_{0}\right) \cos \theta\right)=0,
$$

ce qui nous ramène à la remarque précédente en faisant

$$
\varphi=\left(x-x_{0}\right)^{2}+\left(y-y_{0}\right)^{2}, \quad \psi=\left(x-x_{0}\right) \sin \theta-\left(y-y_{0}\right) \cos \theta, \quad P=2 R .
$$

Le résultant des termes de plus haut degré

$$
\begin{gathered}
f^{0}(x, y)=a_{0} x^{m}+a_{1} x^{m-1} y+\ldots+a_{m} y^{m}, \\
x^{2}+y^{2}
\end{gathered}
$$

des polynômes $f, \varphi$ est la quantité

$$
f^{0}(\mathrm{I}, i) f^{0}(\mathrm{I},-i)=\left(a_{0}-a_{2}+a_{4}-\ldots\right)^{2}+\left(a_{1}-a_{3}+a_{5}-\ldots\right)^{2}
$$

que nous supposons égale à I ; de sorte que $P$ est le résultant des trois polynômes $f, \varphi-2 R \psi, \phi$, ou, ce qui revient au même, $f, \varphi, \psi$. Nous pouvons évaluer ce dernier en formant le produit des valeurs de $\left(x-x_{0}\right)^{2}+\left(y-y_{0}\right)^{2}$ aux points $f=0, \psi=0$, lequel s'obtient aisément en posant $x-x_{0}=\rho \cos \theta, y-y_{0}=\rho \sin \theta$, et a la valeur

$$
\left[\frac{f_{0}}{f^{0}(\cos \theta, \sin \theta)}\right]^{2} \text {. }
$$

Comme le résultant des termes de plus haut degré des polynômes $f, \phi$ est précisément $f^{\circ}(\cos \theta, \sin \theta)$, on trouve bien le résultat annoncé.

Une méthode semblable peut s'appliquer aux deux produits

$$
\Pi\left[\left(x-x_{0}\right) \pm i\left(y-y_{0}\right)\right]
$$

relatifs aux points d'intersection de la droite et du cercle et, par le quotient de ces deux produits, ${ }^{1}$ conduit au second théorème de Laguerre: L'orientation du système des droites qui vont du point $O$ aux points d'intersection est la même que celle des asymptotes de la courbe $f=0$.

'On sait que l'orientation $\theta$ du système des droites allant de l'origine aux points $x^{(h)}, y^{(h)}$, c'est à dire la somme des angles que font ces droites avec l'axe des $x$, est donnée par la formule

$$
e^{2 i \theta}=\prod_{k} \frac{x^{(h)}+i y^{(h)}}{x^{(h)}-i y^{(h)}}
$$


26. Soit encore à trouver le produit des longueurs des sécantes menées du point $O$ à la courbe $f=0$ de manière à la couper sous un angle donné $V$ et l'orientation du système de ces droites.

Les points $x^{(h)}, y^{(h)}\left(h=\mathrm{1}, 2, \ldots, m^{2}\right)$ scront définis par les équations

$$
f(x, y)=0 \text {, }
$$

$$
\left[\left(x-x_{0}\right) f_{y}^{\prime}-\left(y-y_{0}\right) f_{x}^{\prime}\right] \sin V-\left(x_{0} f_{x}^{\prime}+y_{0} f_{y}^{\prime}+f_{z}^{\prime}\right) \cos V=0
$$

et nous considérerons les produits

$$
\Pi\left[\left(x-x_{0}\right) \pm i\left(y-y_{0}\right)\right] .
$$

Prenons d'abord le résultant $R^{0}$ relatif aux polynômes (23), (24). En décomposant $f^{0}(x, y)$ en facteurs $b^{(k)} x-a^{(k)} y$ et substituant $a^{(k)}, b^{(k)}$ dans les termes de plus haut degré de (24), on atrouve pour ce résultant. (en désignant par $\Delta$ le discriminant de $f^{\circ}$ )

$$
\pm \Delta \sin ^{m} V \prod_{i}\left(a^{(k)^{2}}+b^{(k)^{2}}\right)= \pm \Delta \sin ^{m} V f^{0}(\mathrm{r}, i) f^{0}(\mathbf{1},-i) .
$$

Nous connaissons d'ailleurs le résultant $R^{0}$ pour les équations (23) et

$$
x-x_{0}+i_{1}\left(y-y_{0}\right)=0
$$

lequel est $f^{0}(\mathbf{I}, i)$, de sorte que nous sommes ramenés an calcul du produit des résultats de substitution, dans le premier membre de l'équation (24), des solutions communes à (23) et à (25). Or, moyennant ces dernières, le premier membre de (24) peut s'écrire sous l'une des deux formes

$$
\begin{aligned}
& -\left(x_{0} f_{x}^{\prime}+y_{0} f_{y}^{\prime}+f_{z}^{\prime}\right) e^{i \Gamma}, \\
& -\left(x-x_{0}\right)\left(f_{x}^{\prime}+i f_{y}^{\prime}\right) e^{i V} .
\end{aligned}
$$

Partons de la première forme: elle nous représente, à un facteur près, le résultant des polynômes $f, x-x_{0}+i\left(y-y_{0}\right)$ et $x_{0} f_{x}^{\prime}+y_{0} f_{y}^{\prime}+f_{z}^{\prime}$, c'est à dire le produit $\Pi^{\prime}\left(\left(x-x_{0}\right)+i\left(y-y_{0}\right)\right)$ relatif aux tangentes menćes du point $O$ à la courbe, multiplié par le résultant des termes de plus haut degré des polynômes $f, x_{0} f_{x}^{\prime}+y_{0} f_{y}^{\prime}+f_{z}^{\prime}$, lequel est égal an produit de 
$\frac{\Delta}{\sqrt{\Pi\left(a^{(k)^{2}}+b^{\left.(k)^{2}\right)}\right.}}$ par le produit $\Pi_{d}$ des distances du point $O$ aux asymptotes de $f=o$. Nous obtenons donc

$$
\Pi\left(\left(x-x_{0}\right)+i\left(y-y_{0}\right)\right)=\Pi^{\prime}\left(x-x_{0}+i\left(y-y_{0}\right)\right) \frac{e^{m i V}}{\sin ^{m} V} \sqrt{\frac{f^{0}(\mathrm{I}, i)}{f^{0}(\mathrm{I},-i)}} \Pi_{d} .
$$

La seconde forme (27) se traite par des procédés tout semblables et nous donne

$$
\Pi\left(x-x_{0}+i\left(y-y_{0}\right)\right)=f_{0} \Pi\left(\xi-x_{0}+i\left(\eta-y_{0}\right)\right) \frac{e^{m i V}}{\sin ^{m} V f^{0}(1,-\bar{i})},
$$

$\xi$, $\eta$ désignant les coordonnées des foyers réels de la courbe.

$\mathrm{Si}$, dans ces formules nous changeons $i$ en $-i$, elles nous feront connaître le produit $\Pi\left(x-x_{0}-i\left(y-y_{0}\right)\right)$. En multipliant, nous aurons le produit des longueurs des sécantes, égal au produit des longueurs des tangentes, multiplié par le produit des obliques menés du point $O$ aux asymptôtes et les coupant sous l'angle $V$, ou encore à $f_{o}$ multiplié par le produit des distances du point $O$ aux foyers réels et divisé par $\sin ^{m} V$ (en supposant $\left.f^{0}(\mathrm{I}, i) f^{0}(\mathrm{I},-i)=\mathrm{I}\right)$.

En divisant, au contraire, les deux produits conjugués l'un par l'autre, on a l'orientation du système de sécantes, égale à l'orientation des tangentes, plus celle des asymptôtes, plus $m$ fois l'angle $V$; l'orientation des tangentes étant la même que celle des droites qui vont aux foyers réels.

Ces théorèmes sont établis, il est vrai, dans l'hypothèse où la courbe donnée n'a pas de point double. Mais, dans le cas contraire, les points doubles figureraient avec le même ordre de multiplicité comme extrêmité de tangentes, ou de normales, ou comme foyers. Ils s'élimineraient par conséquent des résultats.

27. D'autres théorèmes du même type ont encore été donnés dans les mémoires précédemment cités. Nous les laissons de côté pour rechercher si les propriétés du résultant nous permettent de calculer des fonctions symétriques autres que des produits.

Il y a tout d'abord lieu d'observer que la remarque donnée dans la première partie, $n^{\circ} 8$, permet de ramener à la recherche d'un résultant l'expression de toute fonction symétrique des solutions communes à plusieurs équations donnée sous forme invariante. 
Nous nous bornerons ici à considérer les fonctions symétriques qui se présentent comme la somme des valeurs que prend une certaine fonction rationnelle aux points communes à plusieurs courbes ou surfaces. Ces quantités se ramènent immédiatement à des produits à l'aide de l'identité

$$
\frac{U}{V}=\frac{d}{d \lambda_{\lambda=0}} \log (V+\lambda U)
$$

Supposons que $U$ et $V$ soient deux polynômes homogènes du mème degré $p$ aux trois variables $x, y, z$, et proposons-nous de calculer la somme des valeurs de $\frac{U}{V}$ aux points d'intersection des deux courbes $f=0, \varphi=0$.

Nous devons admettre tout d'abord que $V$ ne s'annule en aucun de ces points, sans quoi la valeur correspondante de $\frac{U}{V}$ serait, en général, infinie on indéterminée. Dans ces conditions, le produit $n(V+\lambda U)$ étendu aux points $(f=0, \varphi=0)$ se calculera à l'aide du produit $\Pi \varphi$ étendu aux points

$$
(f=0, V+\lambda U=0) \text {. }
$$

De ces derniers, certains seront fixes, ceux qui seront communs aux trois courbes $f=0, U=0, V=0$. Ils donneront dans notre produit des facteurs indépendants de $\lambda$, lesquels ne formeront aucun terme à la dérivée logarithmique. Nous n'aurons donc à considérer que les points d'intersection (29) mobiles, lesquels seront, pour $\lambda$ très petit, infiniment voisins des intersections de $f=0$ avec $V=0$, mais non confondus avec elles. En particulier, nous n'aurons pas à faire entrer en ligne de compte les points ou $V$ s'annule sans que $\frac{U}{V}$ soit infini.

Considérons donc un cycle de la courbe $f=0$ sur lequel $\frac{U}{V}$ est infini. Les coordonnées homogènes $x, y, z$ seront données par des séries ordonnées suivant les puissances croissantes d'une variable $t$, nulle à l'origine du cycle. Soient alors

$$
\begin{gathered}
\frac{U}{V}=-\frac{1}{\lambda}=t^{-k}(A+B t+\ldots)=\frac{1}{\psi(t)}, \\
\frac{d \log \varphi}{d t}=a+b t+\ldots
\end{gathered}
$$


La courbe $V+\lambda U=0$ aura, pour $\lambda$ très petit, $k$ points d'intersection avec notre cycle infiniment voisins de son origine, et correspondant aux $k$ racines infiniment petites de l'équation (30).

Supposons d'abord, pour simplifier, $k=1$. Alors la courbe $V+\lambda U=0$ coupera le cycle en un point $\mu$ dont le paramètre $t$ aura pour valeur principale $t=-\lambda A$, de sorte qu'on a

$$
\frac{d \log \varphi_{\mu}}{d \lambda}=\frac{d \log \varphi_{\mu}}{d t_{\mu}} \frac{d t_{\mu}}{d \lambda}=-a A+\ldots
$$

Donc le cycle en question fournira à la quantité $\frac{d \Sigma \log \varphi}{d \lambda}$ un terme égal à $-a A$. c'est à dire au coefficient de $\frac{\mathbf{I}}{\mathbf{t}}$ dans le développement de $-\frac{U}{V} \frac{d \log \varphi}{d t}$, ou à l'intégrale $-\frac{\mathrm{I}}{2 i \pi} \int \frac{U}{V} d \log \varphi$ prise le long d'un contour infiniment petit entourant le point analytique origine $\mathrm{du}$ cycle.

La même conclusion subsiste pour une valeur quelconque de $k$. C'est ce qui peut se voir en traçant dans le plan de la variable $t$ un cercle de petit rayon autour du point $t=0$. La somme des .valeurs de $\frac{d \log \varphi}{d \lambda}=-\frac{\frac{d \log \varphi}{d t}}{\frac{d \varphi}{d t}}$ aux points racines de l'équation (30) situés à l'intérieur de ce cercle (et, si le cercle a été pris une fois pour toutes, les $k$ points racines infiniment voisins de l'origine seront à son intérieur pour $\lambda$ suffisamment petit) sera l'intégrale $-\frac{\mathrm{I}}{2 i \pi} \int \frac{\mathrm{I}}{\phi(t)+\lambda} \frac{d \log \varphi}{d t} d t$ prise le long du cercle, laquelle, pour $\lambda=0$, donnera bien $\int \frac{U}{V} d \log \varphi$.

Nous constaterons le mêrne fait directement en posant $\lambda=\nu^{k}$. $t$ est alors une série en $\mu$ commençant par un terme du premier degré. Les $k$ valeurs infiniment petites de $t$ s'obtiennent en multipliant $\mu$ par les différentes racines $k^{\text {èmes }}$ de l'unité. Si d'autre part on a

$$
F(t)=\frac{\mathrm{I}}{\phi(t)} \frac{d \log \varphi}{d t}=\frac{\alpha_{-k}}{t^{k}}+\frac{\alpha_{-k+1}}{t^{k-1}}+\ldots+\frac{\alpha_{-1}}{t}+\alpha_{0}+\alpha_{1} t+\ldots,
$$


il viendra

$$
\begin{gathered}
\frac{d \log \varphi}{d \lambda}=-\frac{\mathrm{I}}{\psi(t)} \frac{d \log \varphi}{d t} \frac{d t}{d \log \lambda} \\
=\frac{\nu}{l_{i}} \frac{d t}{d \nu}\left(\frac{\alpha_{-k}}{t^{k}}+\frac{\alpha_{-k+1}}{t^{k-1}}+\ldots+\frac{\alpha_{-1}}{t}+\alpha_{0}+\alpha_{6} t+\ldots\right) .
\end{gathered}
$$

Or tous les termes de $F(t)$, autres que $\frac{\alpha_{-1}}{t}$, sont des dérivées de fonctions rationnelles de $t$, et par suite, après multiplication par $\frac{\nu}{k} \frac{d t}{d \nu}$, ne donneront pas de termes indépendants de $\nu$. Ils s'élimineront donc, d'après les propriétés de l'équation binóme, lorsqu'on fera la somme des valeurs que prend l'expression $(3 \mathrm{I})$ en $\mathrm{y}$ multipliant $\nu$ successivement par les différentes racines $k^{\text {ème }}$ de l'unité. Reste le seul terme $\frac{\alpha_{-1}}{t}$, qui donnera $\alpha_{-1}$; d'où la conclusion annoncée.

28. Nous évaluons ainsi la dérivée lograrithmique par rapport à $\lambda$ $\mathrm{du}$ produit des valeurs de $\varphi$ aux points $(f=0, V+\lambda U=0)$. Celui-ci diffère du produit primitif par deux facteurs, une puissance de la quantité le (formule (13), $\mathrm{n}^{\circ} \mathrm{I} 3$, première partic) relative aux polynòmes $f, \varphi$, laquelle est indépendante de $\lambda$ et ne figure pas dans la dérivée logarithmique, et la puissance $n^{\text {ème }}$ ( $n$ étant le degré de $\varphi$ ) de la quantité analogue relative aux polynómes $f, V+\lambda U$. Cette dernière donnera un terme $n C$, où $C$ est indépendant de $\varphi$. Nous arrivons donc à la formule finale

$$
\sum \frac{U}{V}=-\mathrm{SH} \frac{U}{V} \frac{d \log \varphi}{d t}+n C
$$

où le signe $\Sigma$ est relatif aux points $(f=0, \varphi=0)$, le signe $S$ aux cycles de $f=0$ sur Lesquels $\frac{U}{V}$ est infini; le symbole $\mathrm{H} \frac{U}{V} \frac{d \log \varphi}{d t}$ désignant le résidu de $\frac{U}{V} d \log \varphi$, c'est à dire le coefficient de $\frac{\mathrm{I}}{t}$ dans le développement de $\frac{U}{V} \frac{d \log \varphi}{d t}$.

Dans le cas le plus simple, où les cycles qui interviennent dans le 
second membre sont tous ordinaires, $V$ ayant aux origines de ces cycles des zéros simples, cette formule s'écrit

$$
\sum \frac{U}{V}=-S \frac{U}{\varphi} \frac{D(f, \varphi)}{D(f, V)}+n C
$$

ò̀ $D(P, Q)$ désigne le déterminant fonctionnel des fonctions $P, Q$ par rapport aux variables $x, y$. Elle correspond, au fond, à la formule (I 3 ) du premier mémoire de M. Humbent sur le théorème d'Abes. ${ }^{1}$

29. Il n'y a pas lieu de chercher une interprétation géométrique pour la constante $C$, car celle-ci dépend du facteur de proportionnalité qui figure dans les coordonnées homogènes $x, y, z$. Ce facteur est effectivement une série en $t$ qui donne un terme dans la dérivée $\frac{d \log \varphi}{d t}$. Mais nous pouvons obtenir une expression simple de $C$ en faisant $\varphi=V$. Nous voyons alors que la formule (32) peut s'écrire

$$
\sum \frac{U}{V}=-\mathrm{SH}_{\bar{V}} \frac{d\left(\log \varphi-\frac{n}{p} \log U\right)}{d t}
$$

( $p$ étant le degré commun de $U$ et de $V$ ).

Ce procédé de détermination de la constante $C$, reposant uniquement sur la disparition du premier membre de (32) lorsque $\varphi=U$, peut être appliqué plus simplement si $U$ se décompose en facteurs. On pourra alors évaluer $C$ en remplaçant $\varphi$ par l'un de ces facteurs. ${ }^{2}$

En particulier, si $\frac{U}{V}$ s'annulle à l'infini, on annulera $C$ en représentant les cycles du second membre en coordonnées absolues $(z=\mathrm{I})$. C'est, par exemple, le cas du théorème de $\mathrm{J}_{\mathrm{ACOBI}}$, où $V$ est le déterminant fonctionnel $D(f, \varphi)$. Comme on a

$$
\frac{d x}{f^{\prime} y}=\frac{d y}{-f^{\prime} x}=-\frac{d \varphi}{D(f, \varphi)}
$$

'Journal de Mathématiques, 4 érie, tome 3, page 346.

2 Dans le cas où la courbe est représentée à l'aide des fonctions fuchsiennes ainsi que le fait M. Humbers, on voit aisément que la constante $C$ est égale à la somme des intégrales $\frac{\mathrm{I}}{2 i \pi} \int \frac{U}{V} d \log (\gamma t+\delta)$ étendues aux différents côtés du polynôme fuchsien, $\left(t, \frac{a t+\beta}{\gamma t+\delta}\right)$ désignant la substitution correspondant à chaque côté. 
la différentielle $\frac{U}{V} d \log \varphi$ se réduit à $-\frac{U}{\varphi} \frac{d x}{f} y$ aux points $V=0$ sauf si ces points sont des points multiples, ce que l'on peut toujours éviter en remplaçant $f$ par $f+h \varphi$ (ce qui ne change pas $D(f, \varphi)$ ). La courbe $f+h \varphi=0$ n'a en effet pour $h$ quelconque, aucun point double, $\dot{a}$ moins que les courbes $f=0, \varphi=0$ n'aient un point double commun ou une branche entière commune, ce qui est exclu par la nature mème de la question. Si donc $V$ est de degré inférieur à $D(f, \hat{\varphi})$, ce qui entraine $C=0$, la formule $\left(32^{\prime}\right)$ nous donne bien $\sum \frac{U}{D(f, \varphi)}=0$.

30. On peut se demander par quoi peuvent être remplacées les résultats précédents lorsque $V$ s'annulle en un ou plusieurs points communs aux courbes $f=0, \varphi=0$.

A cet effet, on remplacera la courbe $\varphi$ par une courbe voisine $\varphi_{1}$ ne passant pas par les points où $V=0$, et on fera tendre $\varphi_{1}$ vers $\varphi$. Si d'abord on considère un cycle de $f=0$ où $V$ s'annulle, mais sans que $\frac{U}{V}$ devienne infini, on voit immédiatement par ce procédé que l'on doit prendre pour valeur de $\frac{U}{V}$, dans le premier membre de la formule (32), la vraie valeur de cette quantité sur le cycle.

Supposons maintenant que la courbe $\varphi=0$ passe par un ou plusieurs points de la courbe $f=0$ ou $\frac{U}{V}$ soit infini et soit $C$ un cycle ayant pour origine tel point. La courbe auxiliaire $\varphi_{1}=0$ coupera la courbe $f=0$ en un certain nombre de points $P^{\prime}$ situés sur les cycles $C$ et en des points $P^{\prime \prime}$ non situés sur ces cycles. Nous inettrons à part, dans le premier membre de la formule (32), les termes qui se rapportent aux points $P^{\prime}$ et nous les ferons passer dans le second nembre pour les joindre aux termes $\mathrm{H}_{\mathcal{V}} \frac{U}{V} \frac{d \log \varphi_{1}}{d t}$ relatifs aux cycles $C$ correspondants.

Soit

$$
\frac{U}{V}=\frac{a_{-k}}{t^{k}}+\frac{a_{-k+1}}{t^{k-1}}+\ldots+\frac{a_{-1}}{t}+a_{0}+a_{1} t+\ldots
$$

le développement de $\frac{U}{V}$ sur un cycle $C$; ce cycle est coupé par la courbe 
$\varphi_{1}=\mathrm{o}$ en $\mu$ points $t_{1}, t_{2}, \ldots, t_{\mu}$, les paramètres $t_{1}, t_{2}, \ldots, t_{\mu}$ tendent vers o lorsque $\varphi_{1}$ tend vers $\varphi$; de sorte qu'on a

$$
\begin{aligned}
& \frac{d}{d t} \log \varphi=-\frac{\mu}{t}+P(t), \\
& \frac{d}{d t} \log \varphi_{1}=-\frac{\mathrm{I}}{t-t_{1}}-\frac{\mathrm{I}}{t-t_{2}}-\ldots-\frac{\mathrm{I}}{t-t_{\mu}}+P_{1}(t),
\end{aligned}
$$

$P(t), P_{1}(t)$ étant deux développements en $t$ dont le second a pour limite le premier. Le cycle $C$ fournira à la formule (32), modifiée comme nous l'avons dit tout à l'heure, le terme

$$
\begin{aligned}
& \mathrm{H}\left[\left(\frac{a_{-k}}{t^{k}}+\frac{a_{-k+1}}{t^{k-1}}+\ldots\right)\left(-\frac{1}{t-t_{1}}-\frac{1}{t-t_{2}}-\ldots-\frac{1}{t-t_{\mu}}+P_{1}(t)\right)\right] \\
& -\sum_{i=1}^{\mu}\left(\frac{a_{-k}}{t_{i}^{k}}+\frac{a_{-k+1}}{t_{i}^{k-1}}+\ldots\right) \\
= & \mathrm{H}\left[\left(\frac{a_{-k}}{t^{k}}+\frac{a_{-k+1}}{t^{k-1}}+\ldots+\frac{a_{-1}}{t}+a_{0}+a_{1}+\ldots\right) P_{1}(t)\right]-\mu P_{1}(\mathrm{o}),
\end{aligned}
$$

car on a, pour $h<\mathrm{o}$,

$$
\mathrm{H}_{\frac{a_{h}}{t} t^{h}}=-a_{h} t_{i}^{h}
$$

Lorsque $\varphi_{1}$ tend vers $\varphi$, cette quantité (33) a pour limite

$$
\mathrm{H}\left[\left(\frac{a_{-k}}{t^{k}}+\frac{a_{-k+1}}{t^{k-1}}+\ldots\right) P(t)\right]-\mu P(\mathrm{o})=\mathbb{H}\left(\frac{a_{-k}}{t^{k}}+\ldots\right) \frac{d \log \varphi}{d t}
$$

On arriverait d'ailleurs au même résultat en intégrant la quantité $\frac{U}{V} \frac{d \log \varphi_{1}}{d t}$ le long d'un contour renfermant les points $o, t_{1}, t_{2}, \ldots, t_{\mu}$.

Ainsi la formule (32) fait connaître la somme des valeurs de $\frac{U}{V}$ aux points communs aux courbes $f=0, \varphi=0$ où cette quantité a une valeur finie.

3I. Soit maintenant $d I$ une différentielle abélienne prise sur la courbe $f=0$. Coupons cette courbe par deux autres de même degré $n$

$$
F=\text { o, } \quad \Phi=\text { 。 }
$$


et cherchons à calculer la somme des intégrales $\int d I$ entre les points d'intersection de $F$ et ceux de $\phi$. Nous poserons à cet effet

$$
F=\psi-u_{1} \chi, \quad \Phi=\psi-u_{2} \chi
$$

de sorte que $\chi=0$ sera une courbe quelconque du faisceau $(\boldsymbol{F}, \Phi)$, et nous considérerons la courbe variable

$$
\phi-u \chi=0
$$

qui coupera $f$ en un certain nombre de points fixes ou mobiles. L'élément d'intégrale $\int \Sigma d I$ pris entre les points $(f=0, \varphi-u \chi=0)$ et $(f=0, \phi-(u+d u) \chi)$ sera

$$
U d u=d u \sum \frac{d I}{d\left(\frac{\phi}{\chi}\right)}
$$

la sommation étant relative aux points d'intersection mobiles des courbes $f=0, \phi-u \chi=0$. Or la somme des valeurs de $\frac{d I}{d\left(\frac{\phi^{\prime}}{\chi}\right)}$ en tous les points $(f=0, \phi-u \chi=0)$, sauf ceux où cette quantité est infinie (lesquels appartiennent, bien entendu aux points d'intersection fixes) est égale à $n C$, plus la somme des résidus, par rapport aux cycles de $f$ où $\frac{d I}{d \frac{\varphi}{\gamma}}$ est infini, de la quantité

$$
-\frac{d I}{d\left(\frac{\phi^{\prime}}{\chi}\right)} \frac{d \log (\psi-u \chi)}{d t}=\frac{-I^{\prime} \chi^{2}}{\psi^{\prime} \chi-\psi^{\prime} \chi} \frac{\psi^{\prime}-u \chi}{\psi^{\prime}-u \chi}
$$

en désignant par $I^{\prime}, \psi^{\prime}$, etc., les dérivées $\frac{d I}{d t}, \frac{d \xi^{\prime}}{d t}$, etc.

$$
\sum \frac{d I}{a\left(\frac{\psi}{\chi}\right)}=\mathrm{SH}\left(\frac{-I^{\prime} \chi^{2}}{\psi^{\prime} \chi-\psi_{\chi}^{\prime}} \frac{\phi^{\prime}-n \gamma^{\prime}}{\psi-u \chi}\right)+n C
$$


De la fraction $\frac{\psi^{\prime}-u \chi^{\prime}}{\psi-u \chi}$ nous retrancherons le terme $\frac{\chi^{\prime}}{\chi}$. On a

$$
\left.\mathrm{SH} \frac{-I^{\prime} \chi^{2}}{\psi^{\prime} \chi-\phi \chi^{\prime}} \frac{\chi^{\prime}}{\chi}\right)+n C=\sum^{\prime} \frac{d I}{d\left(\frac{\phi}{\chi}\right)}
$$

la somme $\Sigma^{\prime}$ étant relative aux points $(f=0, \chi=0)$ où $\frac{d I}{d\left(\frac{\phi}{\gamma}\right)}$ est fini.

Parmi ceux-ci figurent les points communs à la courbe $f$ et au faisceau $(\psi, \chi)$ : ces points disparaitront done dans la soustraction des formules (35), (36). Mais la quantité $\frac{d I}{d\left(\frac{\psi}{\chi}\right)}$ est nulle pour $\chi=0, \phi \neq 0$, à moins que $\chi d I$ ne soit infini, hypothèse que nous pouvons écarter, puisque $\chi=0$ est une courbe arbitraire du faisceau $(F, \Phi)$. Il viendra en conséquence pour le coefficient de du dans l'élément d'intégrale (34)

$$
U=S \mathrm{H}\left(-\frac{\mathrm{I}}{\frac{\varphi}{\chi}-u} \frac{d I}{d t}\right)
$$

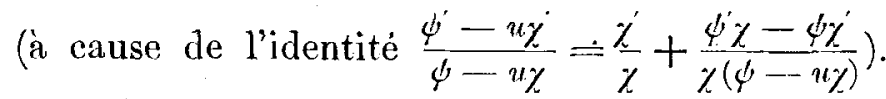

Cette formule (37) n'est autre que la formule fondamentale (3) du mémoire de M. Humbert. ${ }^{1}$ A cause de la disparition du facteur $\phi^{\prime} \chi-\phi \chi^{\prime}$, la somme du second membre sétend aux seuls cycles de $f$ où $\frac{d I}{d t}$ devient infini.

32. Nous venons d'obtenir le coefficient de $d u$; mais rien n'est plus aisé que d'obtenir l'intégrale elle-même. Il est clair en effet que dans la formule (37) nous avons le droit d'intégrer par rapport à $u$ sous le signe $\mathrm{E}$, ce qui nous donne (en intégrant de $u_{1}$ à $u_{2}$ )

$$
\Sigma I=-\$ \mathrm{E}\left(\frac{d I}{d t} \log \frac{F}{\Phi}\right)
$$

\footnotetext{
1 Journal de Mathématiques, $4^{\mathrm{e}}$ série, tome 3, page 334.
} 
33. Il est à remarquer que nous n'aurions point pu poser $\psi=F$, $\chi=\Phi$ et considérer la courbe $F-u \Phi=0, u$ variant de o à $\infty$; car l'intégrale $\int \frac{\frac{d I}{d t}}{\frac{\phi}{\chi}-u} d u$ est infinie avec $u$. On se convainc aisément que les termes infinis disparaissent dans la sommation. C'est ce qui explique comment cette difficulté a pu être écartée par l'artifice simple dont nous nous sommes servis.

34. Nous nous sommes jusqu'ici occupés de courbes planes. Mais tout ce que nous venons de dire s'applique mot pour mot aux courbes gauches.

On considérera alors l'intersection des trois surfaces $f_{1}=0, f_{2}=0$, $\varphi=o$ et l'on obtiendra pour la somme des valeurs de $\frac{U}{V}$ en ces points une formule toute semblable ì la formule (32), et où figureront les cycles de la courbe $\left(f_{1}=0, f_{2}=0\right)$ où $\frac{U}{V}$ devient infinie. (En particulicr on déduira de cette formule le théorème de . $\mathrm{ACOBI}$ généralisé an cas de trois équations.)

Cette formule, établie pour les courbes qui sont intersection complète, s'étend immédiatement aux courbes gauches algébriques quelconques puisque toute pareille courbe devient une intersection complète lorsqu'on lui adjoint une ou plusieurs droites, auxquelles la proposition est applicable.

On pourra en particulier généraliser le théorème de Jaconi à l'intersection d'une courbe gauche algébrique quelconque et d'une surface.

La formule (32) étant une fois démontrée, les déductions qui suivent subsistent sans modification et il est clair que l'on pourrait raisonner de même pour les courbes algébriques définies dans une espace à un nombre quelconque de dimensions.

35. Nous n'avons pas à développer les nombreuses applications des formules (32), (37) et (38), lesquelles ont été traités dans les mémoires précédemment cités de M. HumberT. Nous dirons simplement un mot d'un cas sur lequel ce géomètre n'a pas insisté spécialement, celui des intégrales prises le long d'une courbe fermée. Dans ce cas, la quantité 
$\log \frac{F}{\phi}$ de la formule (38) devra évidemment être remplacée par un multiple de $2 i \pi$.

Soit, par exemple, une courbe $T$ de degré $2 m$ composée de $m$ parties fermées $T_{1}, \Gamma_{2}, \ldots, T_{n}$ à l'intérieur desquelles peut passer une même droite. Par cette droite on pourra faire passer un plan mobile qui coupera chaque branche fermée en deux points. Lorsque notre plan aura accompli une demi-revolution, les points mobiles auront à eux deux parcouru la branche entière sur laquelle ils se trouvent, dans un sens déterminé.

Soit maintenant $d I$ une différentielle abélienne qui ne devient infinie en aucun point réel de notre courbe: les formules précédentes nous permettront de calculer la soimme $S$ des intégrales $\int d I$ prises le long des $m$ branches.

Prenons pour axe des $x$ la droite $D$. Pour éviter un inconvénient analogue à celui que nous avons signalé au $n^{\circ} 33$, nous prendrons notre plan mobile sous la forme

$$
y \sin \theta-z \cos \theta=0,
$$

où $\theta$ variera dans un intervalle égal à $\pi$. On aura alors pour élément d'intégrale

$$
d \theta \sum \frac{d I\left(y^{2}+z^{2}\right)}{y d z-z d y}=d \theta\left[\mathrm{SH} \frac{I^{\prime}\left(y^{2}+z^{2}\right)}{y z^{\prime}-z y^{\prime}} \frac{y^{\prime} \sin \theta-z^{\prime} \cos \theta}{y \sin \theta-z \cos \theta}+C\right]
$$

Nous avons donc à intégrer la différentielle $d \theta \frac{y^{\prime} \sin \theta-z^{\prime} \cos \theta}{y \sin \theta-z \cos \theta}$ dans un intervalle égal à $\pi$ ou en posant $\operatorname{tg} \theta=u$, ’̇ intégrer

$$
\int_{-\infty}^{+\infty} \frac{y^{\prime} u-z^{\prime}}{y u-z} \frac{d u}{\mathrm{I}+u^{2}} .
$$

Cette intégrale, au facteur $2 i \pi$ près, s'obtient en prenant le résidu de la quantité sous le signe $\int$ par rapport au point $u=i$ et $y$ ajoutant le 
résidu relatif au point $u=\frac{z}{y}$ si le coefficient de $i$ dans cette dernière quantité est positif. Le résidu relatif à $u=i$ fournit à l'intégrale de la quantité (39) le terme

$$
\pi \mathrm{SH} \frac{d l\left(y^{2}+z^{2}\right)}{y d z-z d y} \frac{d}{d t} \log (y+i z)
$$

lequel, ajouté à la quantité $C \pi$ provenant de l'intégration du teirme constant $C$, donne une somme égale à

$$
\pi \sum_{\Gamma, y+i z=0} \frac{d I\left(y^{2}+z^{2}\right)}{y d z-z d y}=-0 .
$$

Le résidu de l'intégrale (40), relatif au point $u=\frac{z}{y}$, étant $\frac{y^{\prime} z-z^{\prime} y}{y^{2}+z^{2}}$, on voit qu'il vient simplement

$$
\mathrm{S}=2 i \pi \mathrm{S}^{\prime} \mathrm{K} \frac{d I}{d t}
$$

la somme $S$ ' étant étendue aux infinis de l'intégrale tels que le coefficient de $i$ dans le rapport $\frac{z}{y}$ en ces points soit positif.

L'intégrale $I$ devient infinie en $2 \mu$ points $P_{1}, Q_{1}, P_{2}, Q_{2}, \ldots, P_{\mu}, Q_{\mu}$, imaginaires conjugués deux à deux. On voit que l'on doit faire entrer dans la formule (4I) le résidu relatif à un des points de chaque couple, les points $P_{1}, P_{2}, \ldots, P_{\mu}$ par exemple.

36. La droite $D$ n'est assujettic qu'à la condition de traverser chacune des parties formées $\Gamma_{1}, \Gamma_{2}, \ldots, \Gamma_{m}$ de $I$. Si donc $R$ est une région continue de l'espace, de chaque point de laquelle on voit ces courbes fermées sous de cònes ayant à leur intérieur une partie commune (région limitée par des portions de développables circonscrites à ces courbes), la droite $D$ pourra prendre une position quelconque à l'intérieur de cette région.

Il est clair à priori que le choix des points $P_{1}, P_{2}, \ldots, P_{\mu}$ ne doit pas être influencé par une semblable variation de position de $D$. C'est ce que l'on constate de la manière suivante. 
Soient $\alpha, \beta, \gamma, L, M, N$ les coordonnées plückériennes de $D$, rapportées à des axes quelconques. Comme dans le numéro précédent on suppose qu'il a été choisi un sens sur $D$, ces coordonnées doivent ètre regardées comme définies à un même facteur positif près. Les anciennes coordonnées $y, z$ seront remplacées par des polynômes du premier degré $a x+b y+c z+d t, a^{\prime} x+b^{\prime} y+c^{\prime} z+d^{\prime} t$ tels que les déterminants $b c^{\prime}-c b^{\prime}, a c^{\prime}-c a^{\prime}, \ldots$ soient proportionnelles, avec un facteur de proportionnalité positif, aux quantités $\alpha, \beta$, etc.

Considérons d'autre part un couple de points $P_{1}, Q_{1}$ imaginaires conjuguées, situés à distance finie ou à l'infini, dont les coordonnées homogènes sont $x \pm x^{\prime} i, y \pm y^{\prime} i, z \pm z^{\prime} i, t \pm t^{\prime} i$. Par ces deux points passe une droite réelle $D_{1}$, et au choix de l'un des points $P_{1}, Q_{1}$ par opposition à l'autre correspondra le choix d'un sens sur cette droite: le sens positif par rapport auquel le segment qui va du point choisi à l'autre aura la partie imaginaire positive. On obtiendra les coordonnées de la droite $D_{1}$, prise dans le sens en question (si l'on a choisi le point

$$
P_{1}\left(x+x^{\prime} i, y+y^{\prime} i, z+z^{\prime} i, t+t^{\prime} i\right)
$$

par opposition au point $Q_{1}\left(x-x^{\prime} i, y-y^{\prime} i, z-z^{\prime} i, t-t^{\prime} i\right)$,) en divisant par $i$ les déterminants $\left(x+x^{\prime} i\right)\left(t-t^{\prime} i\right)-\left(x-x^{\prime} i\right)\left(t+t^{\prime} i\right)$, etc. ${ }^{1}$

Le coefficient de $i$ dans le rapport

$$
\frac{a^{\prime}\left(x+x^{\prime} i\right)+b^{\prime}\left(y+y^{\prime} i\right)+c^{\prime}\left(z+z^{\prime} i\right)+d^{\prime}\left(t+t^{\prime} i\right)}{a\left(x+x^{\prime} i\right)+b\left(y+y^{\prime} i\right)+c\left(z+z^{\prime} i\right)+d\left(t+t^{\prime} i\right)}
$$

est égal, à un dénominateur positif près, à l'expression

$$
\alpha L_{1}+\beta M_{1}+\gamma N_{1}+\alpha_{1} L+\beta_{1} M+\gamma_{1} N
$$

expression bien connue dont le signe dépend du sens de rotation d'une des semi-droites $D, D_{1}$ par rapport à l'autre. C'est donc ce sens de rotation qui décidera du choix du point $P_{1}$.

1 En effet cette conclusion est vraie pour $t \pm t^{\prime} i=1$, et d'autre part, les signes des déterminants ne sont pas altérés si on multiplie les quatre coordonnées des deux points $P_{1}, Q_{1}$ par deux quantités imaginaires conjuguées l'une de l'autre. 
D'ailleurs ce sens de rotation ne varie pas par le déplacement de $D$ à l'intérieur de la région $R$, car la quantité (42) ne devient nulle que si les droites $D, D_{1}$ sont dans un mème plan: ce qui ne saurait arriver, puisque ce plan couperait la courbe $I$, de degré $2 m$, en $2 m$ points réels et 2 imaginaires. 